\title{
ON THE INDEX-CONJECTURE OF LENGTH FOUR MINIMAL ZERO-SUM SEQUENCES II
}

\author{
CAIXIA SHEN AND LI-MENG XIA ${ }^{\dagger}$
}

Faculty of Science, Jiangsu University

Zhenjiang, 212013, Jiangsu Province, P.R. China

\begin{abstract}
Let $G$ be a finite cyclic group. Every sequence $S$ over $G$ can be written in the form $S=\left(n_{1} g\right) \cdot \ldots \cdot\left(n_{l} g\right)$ where $g \in G$ and $n_{1}, \cdots, n_{l} \in[1, \operatorname{ord}(g)]$, and the index ind $S$ of $S$ is defined to be the minimum of $\left(n_{1}+\cdots+n_{l}\right) / \operatorname{ord}(g)$ over all possible $g \in G$ such that $\langle g\rangle=G$. A conjecture says that if $G$ is finite such that $\operatorname{gcd}(|G|, 6)=1$, then $\operatorname{ind}(S)=1$ for every minimal zero-sum sequence $S$. In this paper, we prove that the conjecture holds if $S$ is reduced and the (A1) condition is satisfied(see [19]).
\end{abstract}

Key Words: cyclic group, minimal zero-sum sequence, index of sequences, reduced.

2000 Mathematics Subject Classification: 11B30, 11B50, 20K01

\section{INTRODUCTION}

Throughout the paper, let $G$ be an additively written finite cyclic group of order $|G|=n$. By a sequence over $G$ we mean a finite sequence of terms from $G$ which is unordered and repetition of terms is allowed. We view sequences over $G$ as elements of the free abelian monoid $\mathcal{F}(G)$ and use multiplicative notation. Thus a sequence $S$ of length $|S|=k$ is written in the form $S=\left(n_{1} g\right) \cdot \ldots \cdot\left(n_{k} g\right)$, where $n_{1}, \cdots, n_{k} \in \mathbb{N}$ and $g \in G$. We call $S$ a zero-sum sequence if $\sum_{j=1}^{k} n_{j} g=0$. If $S$ is a zero-sum sequence, but no proper nontrivial subsequence of $S$ has sum zero, then $S$ is called a minimal zero-sum sequence. Recall that the index of a sequence $S$ over $G$ is defined as follows.

Definition 1.1. For a sequence over $G$

$$
S=\left(n_{1} g\right) \cdot \ldots \cdot\left(n_{k} g\right), \quad \text { where } 1 \leq n_{1}, \cdots, n_{k} \leq n,
$$

the index of $S$ is defined by $\operatorname{ind}(S)=\min \left\{\|S\|_{g} \mid g \in G\right.$ with $\left.\langle g\rangle=G\right\}$, where

$$
\|S\|_{g}=\frac{n_{1}+\cdots+n_{k}}{\operatorname{ord}(g)} .
$$

Clearly, $S$ has sum zero if and only if $\operatorname{ind}(S)$ is an integer.

Conjecture 1.2. Let $G$ be a finite cyclic group such that $\operatorname{gcd}(|G|, 6)=1$. Then every minimal zero-sum sequence $S$ over $G$ of length $|S|=4$ has $\operatorname{ind}(S)=1$.

The index of a sequence is a crucial invariant in the investigation of (minimal) zero-sum sequences (resp. of zero-sum free sequences) over cyclic groups. It was first addressed by KleitmanLemke (in the conjecture [9, page 344]), used as a key tool by Geroldinger ([6, page 736]), and then

\footnotetext{
†the corresponding author's email: xialimeng@ujs.edu.cn.

Supported by the NNSF of China (Grant No. 11001110, 11271131).
} 
investigated by Gao [3] in a systematical way. Since then it has received a great deal of attention (see for example $[1,2,4,7,10,11,12,13,14,15,16,17,18]$ ). A main focus of the investigation of index is to determine minimal zero-sum sequences of index 1 . If $S$ is a minimal zero-sum sequence of length $|S|$ such that $|S| \leq 3$ or $|S| \geq\left\lfloor\frac{n}{2}\right\rfloor+2$, then $\operatorname{ind}(S)=1$ (see $[1,14,16]$ ). In contrast to that, it was shown that for each $k$ with $5 \leq k \leq\left\lfloor\frac{n}{2}\right\rfloor+1$, there is a minimal zero-sum subsequence $T$ of length $|T|=k$ with $\operatorname{ind}(T) \geq 2([13,15])$ and that the same is true for $k=4$ and $\operatorname{gcd}(n, 6) \neq 1$ ([13]). The left case leads to the above conjecture.

In [12], it was proved that Conjecture 1.2 holds true if $n$ is a prime power. In [11], it was proved that Conjecture 1.2 holds for $n=p_{1}^{\alpha} \cdot p_{2}^{\beta},\left(p_{1} \neq p_{2}\right)$, and at least one $n_{i}$ co-prime to $|G|$.

In [19], it was proved that Conjecture 1.2 holds if the sequence $S$ is reduced and at least one $n_{i}$ co-prime to $|G|$.

By the result of [19], a minimal zero-sum sequence $S=\left(x_{1} g\right) \cdot\left(x_{2} g\right) \cdot\left(x_{3} g\right) \cdot\left(x_{4} g\right)$ over $G$ is reduced then $|G|$ has at most two prime factors or one of the following holds:

(A1) $\left\{\operatorname{gcd}\left(x_{i}, n\right) \mid i=1,2,3,4\right\}=\left\{p_{1}, p_{2}, p_{1} p_{3}, p_{2} p_{3}\right\}$;

(A2) $\left\{\operatorname{gcd}\left(x_{i}, n\right) \mid i=1,2,3,4\right\}=\left\{1, p_{1}, p_{2}, p_{1} p_{2}\right\}$;

(A3) $\operatorname{gcd}\left(x_{i}, n\right)=1$ for $i=1,2,3,4$;

(A4) $\operatorname{gcd}\left(x_{1}, n\right)=1, \operatorname{gcd}\left(x_{2}, n\right)=p_{1} p_{2}, \operatorname{gcd}\left(x_{3}, n\right)=p_{1} p_{3}, \operatorname{gcd}\left(x_{4}, n\right)=p_{2} p_{3}$.

In this paper, we give the affirmative proof under assumption (A1), and our main result can be stated by the following theorem:

Theorem 1.3. Let $G=\langle g\rangle$ be a finite cyclic group such that $|G|=p_{1} p_{2} p_{3}$ and $\operatorname{gcd}(n, 6)=1$. If $S=\left(x_{1} g, x_{2} g, x_{3} g, x_{4} g\right)$ is a minimal zero-sum sequence over $G$ such that

$$
\left\{\operatorname{gcd}\left(n, x_{i}\right) \mid i=1,2,3,4\right\}=\left\{p_{1}, p_{2}, p_{1} p_{3}, p_{2} p_{3}\right\} .
$$

Then $\operatorname{ind}(S)=1$.

It was mentioned in [13] that Conjecture 1.2 was confirmed computationally if $n \leq 1000$. Hence, throughout the paper, we always assume that $n>1000$.

\section{Preliminaries and Renumbering the SEQuence}

Throughout, let $\mathrm{G}$ be a cyclic group of order $|G|=n>1000$. Given real numbers $a, b \in \mathbb{R}$, we use $[a, b]=\{x \in \mathbb{Z} \mid a \leq x \leq b\}$ to denote the set of integers between $a$ and $b$, and similarly, set $[a, b)=\{x \in \mathbb{Z} \mid a \leq x<b\}$. For $x \in \mathbb{Z}$, we denote by $|x|_{n} \in[1, n]$ the integer congruent to $x$ modulo $n$. Suppose that $n$ has a prime decomposition $n=p^{\alpha} q^{\beta}$. Let $S=\left(x_{1} g\right) \cdot \ldots \cdot\left(x_{4} g\right)$ be a minimal zero-sum sequence over $G$ such that $\operatorname{ord}(g)=n=|G|$ and $1 \leq x_{1}, x_{2}, x_{3}, x_{4} \leq n-1$. Then $x_{1}+x_{2}+x_{3}+x_{4}=\nu n$, where $1 \leq \nu \leq 3$.

Let $S$ be the sequence as described in Theorem 1.3. Similar to Remark 2.1 of [11], we may always assume that $x_{1}=e, e+x_{2}+x_{3}+x_{4}=2 n$ and $e<x_{2}<\frac{n}{2}<x_{3} \leq x_{4}<n-e$. Let $c=x_{2}, b=n-x_{3}, a=n-x_{4}$, then it is easy to show that the following proposition implies Theorem 1.3.

Proposition 2.1. Let $n=p_{1} p_{2} p_{3}$, where $p_{1}, p_{2}, p_{3}$ are three different primes, and $\operatorname{gcd}(n, 6)=1$. Let $S=(g) \cdot(c g) \cdot((n-b) g) \cdot((n-a) g)$ be a minimal zero-sum sequence over $G$ such that 
$\operatorname{ord}(g)=|G|=n$, and

$$
\{\operatorname{gcd}(n, e), \operatorname{gcd}(n, c), \operatorname{gcd}(n, b), \operatorname{gcd}(n, a)\}=\left\{p_{1}, p_{2}, p_{1} p_{3}, p_{2} p_{3}\right\},
$$

where $e+c=a+b$. Then $\operatorname{ind}(S)=1$.

Notice that: for convenience, we list two sufficient conditions introduced in Remark 2.1 of [11].

(1) If there exists positive integer $m$ such that $\operatorname{gcd}(n, m)=1$ and $\left|m x_{1}\right|_{n}+\left|m x_{2}\right|_{n}+\left|m x_{3}\right|_{n}+$ $\left|m x_{4}\right|_{n}=3 n$, then $\operatorname{ind}(S)=1$.

(2) If there exists positive integer $m$ such that $\operatorname{gcd}(n, m)=1$ and at most one $\left|m x_{i}\right|_{n} \in\left[1, \frac{n}{2}\right]$ (or, similarly, at most one $\left|m x_{i}\right|_{n} \in\left[\frac{n}{2}, n\right]$ ), then $\operatorname{ind}(S)=1$.

Lemma 2.2. Proposition 2.1 holds if one of the following conditions holds:

(1) There exist positive integers $k, m$ such that $\frac{k n}{c} \leq m \leq \frac{k n}{b}, \operatorname{gcd}(m, n)=1,1 \leq k \leq b$, and $m a<n$.

(2) There exists a positive integer $M \in\left[1, \frac{n}{2 e}\right]$ such that $\operatorname{gcd}(M, n)=1$ and at least two of the following inequalities hold:

$$
|M a|_{n}>\frac{n}{2},|M b|_{n}>\frac{n}{2},|M c|_{n}<\frac{n}{2}
$$

Lemma 2.3. If there exist integers $k$ and $m$ such that $\frac{k n}{c} \leq m \leq \frac{k n}{b}, \operatorname{gcd}(m, n)=1,1 \leq k \leq b$, and $a \leq \frac{b}{k}$, then Proposition 3.1 holds.

From now on, we assume that $s=\left\lfloor\frac{b}{a}\right\rfloor$. Then we have $1 \leq s \leq \frac{b}{a}<s+1$. Since $b \leq \frac{n}{2}$, we have $\frac{n}{2 b}=\frac{(2 s-t) n}{2 b}-\frac{(2 s-t-1) n}{2 b}>1$, and then $\left[\frac{(2 s-t-1) n}{2 b}, \frac{(2 s-t) n}{2 b}\right]$ contains at least one integer for every $t \in[0, s-1]$.

Lemma 2.4. Suppose that $a>2 e, s \geq 2$ and $\left[\frac{(2 s-2 t-1) n}{2 b}, \frac{(s-t) n}{b}\right]$ contains an integer co-prime to $n$ for some $t \in\left[0, \cdots,\left\lfloor\frac{s}{2}\right\rfloor-1\right]$. Then Proposition 2.1 holds.

For the proof of Lemma 3.3, Lemma 3.4 and Lemma 3.5, one is referred to the proof of Lemma 2.3-2.5 in [1], and we omit it here.

Let $\Omega$ denote the set of those integers: $x \in \Omega$ if and only if $x \in\left[\frac{(2 s-t-1) n}{2 b}, \frac{(s-t) n}{b}\right]$ for some $t \in\left[0,\left\lfloor\frac{s}{2}\right\rfloor-1\right]$. By Lemma 3.5, we also assume that

$(B):\left[\frac{(2 s-2 t-1) n}{2 b}, \frac{(s-t) n}{b}\right]$ contains no integers co-prime to $n$ for every $t \in\left[0,\left\lfloor\frac{s}{2}\right\rfloor-1\right]$.

Lemma 2.5. Suppose that $a>2 e, s \geq 2$ and $\left[\frac{(2 s-2 t-1) n}{2 b}, \frac{(s-t) n}{b}\right]$ contains no integers co-prime to $n$ for every $t \in\left[0,\left\lfloor\frac{s}{2}\right\rfloor-1\right]$. Then $\left[\frac{(2 s-t-1) n}{2 b}, \frac{(s-t) n}{b}\right]$ contains at most 3 integers for every $t \in\left[0,\left\lfloor\frac{s}{2}\right\rfloor-1\right]$. Hence $\frac{n}{2 b}<4$.

Lemma 2.6. Suppose that $a>2 e, s \geq 4$ and $\left[\frac{(2 s-2 t-1) n}{2 b}, \frac{(s-t) n}{b}\right]$ contains no integers co-prime to $n$ for every $t \in\left[0,\left\lfloor\frac{s}{2}\right\rfloor-1\right]$. Then $\left[\frac{(2 s-2 t-1) n}{2 b}, \frac{(s-t) n}{b}\right]$ contains at most two integers for every $t \in\left[0,\left\lfloor\frac{s}{2}\right\rfloor-1\right]$ and $\frac{n}{2 b}<3$.

Lemma 2.7. Suppose that $a>2 e$ and $s \geq 6$, then there exists $t_{1} \in\left\{0,\left\lfloor\frac{s}{2}\right\rfloor-1\right\}$ such that $\left[\frac{\left(2 s-t_{1}-1\right) n}{2 b}, \frac{\left(2 s-t_{1}\right) n}{2 b}\right]$ contains exactly one integer and $\frac{n}{2 b}<2$.

Lemma 2.8. Suppose that $a>2 e$ and $s \geq 8$, then $\left[\frac{(2 s-2 t-1) n}{2 b}, \frac{(2 s-t) n}{b}\right]$ contains exactly one integer for every $t \in\left[0,\left\lfloor\frac{s}{2}\right\rfloor-1\right]$. 
Lemma 2.9. Under assumption $a>2 e$, we have $s \leq 9$.

For the proof of Lemma 2.2-2.9 and more details, one is referred to [20], Li and Peng's paper [11] is also recommended.

Out of question, we can assume that $e=\min \left\{p_{1}, p_{2}\right\}$, without less of generality, let $p_{1}<p_{2}$, then $e=p_{1}$.

Lemma 2.10. If $a<4 e$, then $p_{3}<p_{1}=e<p_{2}$ and $a=k p_{2}$ for some $k \in[1,3]$.

Proof. Since $p_{2}>p_{1}$ and $p_{2} p_{3}>p_{1} p_{3} \geq 5 p_{1}$, it must hold that $a=k p_{2}$ for some $k \in[1,3]$. Hence we only need prove the case $p_{3}>p_{1}$.

If $p_{3}>p_{1}$ and $a=3 p_{2}$, it holds that $p_{3} \mid(c-b)=(a-e)=3 p_{2}-p_{1} \in 2 \mathbb{Z}$. If $3 p_{2}-p_{1} \geq 4 p_{3}$, we have $a \geq 4 p_{3}+p_{1}>5 p_{1}=5 e$, a contradiction. Then $3 p_{2}-p_{1}=2 p_{3}$ and $p_{3}>p_{2}$. Simply computing shows that $p_{1} \geq 29$ and $p_{3} \geq 41$. Then $a=3 p_{2}>2 p_{1}=2 e$ and $\frac{b}{a} \geq \frac{p_{1} p_{3}}{3 p_{2}}>\frac{p_{3}}{4}>10$, which contradicts to the results of Lemma 2.9 .

If $p_{3}>p_{1}$ and $a=p_{2}$, it holds that $p_{3} \mid(c-b)=(a-e)=p_{2}-p_{1} \in 2 \mathbb{Z}$. If $p_{2}-p_{1} \geq 4 p_{3}$, we have $a>5 e$, a contradiction. Then $p_{2}-p_{1}=2 p_{3}$. Applying Lemma 2.9, similar to above, we have $b=2 p_{1} p_{3}, p_{1}=11, p_{3}=13, p_{2}=37$, or $b=p_{1} p_{3}$. If $p_{1}=11, p_{3}=13, p_{2}=37$ and $b=2 p_{1} p_{3}$, then $p_{2} p_{3} \mid c=b+a-e=2 p_{1} p_{3}+p_{2}-p_{1}=312<481=p_{2} p_{3}$, a contradiction. If $b=p_{1} p_{3}$, then $p_{2} p_{3} \mid c=b+a-e=p_{1} p_{3}+p_{2}-p_{1} \leq\left(p_{2}-4\right) p_{3}+p_{2}-p_{1}<p_{2} p_{3}$, which is a contradiction.

If $p_{3}>p_{1}$ and $a=2 p_{2}$, it holds that $p_{3} \mid(c-b)=(a-e)=2 p_{2}-p_{1} \in 2 \mathbb{Z}+1$. If $2 p_{2}-p_{1} \geq 3 p_{3}$, we have $a>4 e$, a contradiction. Then $2 p_{2}-p_{1}=p_{3}$. Applying Lemma 2.9, similar to above, we have $b=p_{1} p_{3}$ and $p_{1}=7, p_{2}=13, p_{3}=19$ or $p_{1}=11, p_{2}=17, p_{3}=23$. Since $p_{2} p_{3} \mid c=b+a-e=$ $p_{1}\left(p_{3}-1\right)+p_{2}<p_{3} p_{2}$, we obtain a contradiction.

Lemma 2.11. If $2 e<a<4 e$, then $\operatorname{ind}(S)=1$.

Proof. By Lemma 2.10, it holds that $a=k p_{2}$ for some $k \in[1,3]$. We distinguish three cases according to the value of $k$.

Case 1. $k=1$.

Subcase 1.1. $p_{2} \mid c$.

If $c=p_{2} p_{3}$, then $p_{1} \mid c-a=p_{2}\left(p_{3}-1\right)$, which implies $p_{1} \mid\left(p_{3}-1\right)<p_{1}$, a contradiction.

If $c=3 p_{2} p_{3}$, then $\frac{b}{a}=\frac{c+e-a}{a}>3 p_{3}-1 \geq 14>10$, a contradiction.

If $c=2 p_{2} p_{3}$, then $p_{1} \mid c-a=p_{2}\left(2 p_{3}-1\right)$, which implies that $p_{1}=2 p_{3}-1$. If $p_{3} \geq 7$, we have $\frac{b}{a}=\frac{c+e-a}{a} \geq 13>10$, a contradiction. If $p_{3}=5$, then $p_{1} \mid 9$, a contradiction.

Subcase 1.2. $p_{2} \mid b$.

If $b=p_{2} p_{3}$, then $p_{1} \mid b+a=p_{2}\left(p_{3}+1\right)$, which implies $p_{1}=p_{3}+1<p_{1}$, a contradiction.

If $b \geq 2 p_{2} p_{3}$, then $\frac{b}{a} \geq 10$, a contradiction.

Case 2. $k=2$.

Subcase 2.1. $p_{2} \mid c$.

If $c=p_{2} p_{3}$, then $p_{1} \mid c-a=p_{2}\left(p_{3}-2\right)$, which implies $p_{1} \mid\left(p_{3}-2\right)<p_{1}$, a contradiction.

If $c=2 p_{2} p_{3}$, then $p_{1} \mid c-a=2 p_{2}\left(p_{3}-1\right)$, which implies $p_{1} \mid\left(p_{3}-1\right)<p_{1}$, a contradiction. 
If $c=3 p_{2} p_{3}$, then $p_{1} \mid c-a=p_{2}\left(3 p_{3}-2\right)$. If $p_{3} \geq 11$, we have $\frac{b}{a}=\frac{c+e-a}{a}>10$, a contradiction. If $p_{3}=5$, then $p_{1}=13$ and $p_{2}=19, b=c+e-a=3 \times 19 \times 5+13-38=260$. Since $17<\frac{4 n}{c}=\frac{52}{3}<18<19=\frac{4 n}{b}$ and $\operatorname{gcd}(n, 18)=1,18 a=684<n$, let $m=18$ and $k=4$, then $\operatorname{ind}(S)=1$. If $p_{3}=7$, then $p_{1}=19$. However, we can't find a prime $p_{2}$ such that $7 \mid\left(2 p_{2}-19\right)$ and $19<p_{2}<38$.

\section{Subcase 2.2. $p_{2} \mid b$.}

If $b=p_{2} p_{3}$, then $p_{1} \mid b+a=p_{2}\left(p_{3}+2\right)$, which implies $p_{1}=p_{3}+2$. By Lemma 2.9, we have $p_{3} \leq 19$, in further, $p_{3} \in\{5,11,17\}$. If $p_{3}=5, p_{1}=7, p_{2} \leq 13$, which contradicts to $n=p_{1} p_{2} p_{3}>1000$. If $p_{3}=11, p_{1}=13$, then $p_{2} \in\{17,19\}$, which contradicts to $p_{3} \mid\left(2 p_{2}-p_{1}\right)$. If $p_{3}=17, p_{1}=19$, then $p_{2} \in\{23,29,31,37\}$, which also contradicts to $p_{3} \mid\left(2 p_{2}-p_{1}\right)$.

If $b=2 p_{2} p_{3}$, then $p_{1} \mid b+a=2 p_{2}\left(p_{3}+1\right)$, which implies $p_{1} \mid p_{3}+1<p_{1}$, a contradiction.

If $b=3 p_{2} p_{3}$, then $p_{1} \mid b+a=p_{2}\left(3 p_{3}+2\right)$. If $p_{3} \geq 7$, we have $\frac{b}{a} \geq \frac{23}{2}>10$, a contradiction. If $p_{3}=5$, then $p_{1}=17$ and $p_{2}=31, c=b+a-e=510$. Since $\frac{6 n}{c}=31<32<34=\frac{6 n}{b}$ and $\operatorname{gcd}(n, 32)=1,32 a=1984<2635=n$, let $m=32$ and $k=6$, then $\operatorname{ind}(S)=1$.

Case 3. $k=3$.

Subcase 3.1. $p_{2} \mid c$.

If $c=p_{2} p_{3}$, then $p_{1} \mid c-a=p_{2}\left(p_{3}-3\right)$, which implies $p_{1} \mid\left(p_{3}-2\right)<p_{1}$, a contradiction.

If $c=3 p_{2} p_{3}$, then $p_{1} \mid c-a=3 p_{2}\left(p_{3}-1\right)$, which implies $p_{1} \mid\left(p_{3}-1\right)<p_{1}$, a contradiction.

If $c=2 p_{2} p_{3}$, then $p_{1} \mid c-a=p_{2}\left(2 p_{3}-3\right)$, which implies $p_{1}=2 p_{3}-3$. If $p_{3} \geq 17$, we have $\frac{b}{a}=\frac{c+e-a}{a}>10$, a contradiction. If $p_{3}=5$, then $p_{1}=7$ and $p_{2}<10$, a contradiction. If $p_{3}=7$, then $p_{1}=11$ and $p_{2}=13$, thus $\frac{n}{c}<6<\frac{n}{b}$ and $\operatorname{gcd}(n, 6)=1$. Let $m=6$ and $k=1$, then $\operatorname{ind}(S)=1$. If $p_{3}=11$, then $p_{1}=19$, but there exists no prime $p_{2}$ such that $3 p_{2}<4 p_{1}$ and $p_{3} \mid\left(3 p_{2}-p_{1}\right)$. If $p_{3}=13$, then $p_{1}=23$, there exists no prime $p_{2}$ such that $3 p_{2}<4 p_{1}$ and $p_{3} \mid\left(3 p_{2}-p_{1}\right)$. If $p_{3}=17$, which implies $p_{1} \mid 49$ hence $p_{1}=7<p_{3}$, a contradiction..

Subcase 3.2. $p_{2} \mid b$.

If $b=p_{2} p_{3}$, then $p_{1} \mid b+a=p_{2}\left(p_{3}+3\right)$, which implies $2 p_{1} \mid p_{3}+3$, a contradiction. If $b=3 p_{2} p_{3}$, then $p_{1} \mid b+a=3 p_{2}\left(p_{3}+1\right)$, which implies $p_{1} \mid p_{3}+1<p_{1}$, a contradiction.

If $b=2 p_{2} p_{3}$, then $p_{1} \mid b+a=p_{2}\left(2 p_{3}+3\right)$. If $p_{3} \geq 17$, we have $\frac{b}{a} \geq \frac{37}{3}>10$, a contradiction. If $p_{3}=5$, then $p_{1}=13$ and $p_{2}=17$, which contradicts to $p_{3} \mid\left(3 p_{2}-p_{1}\right)$. If $p_{3}=7$, then $p_{1}=17$ and there exists no suitable $p_{2}$. If $p_{3}=11$, we can't find suitable $p_{1}$. If $p_{3}=13$, then $p_{1}=29$, we can't find suitable $p_{2}$.

Lemma 2.12. If $a<2 e$ and $a \mid b$, then $\operatorname{ind}(S)=1$.

Proof. Let $m=\frac{n+a}{a}, m_{1}=\frac{n+2 a}{a}, m_{2}=\frac{n+3 a}{a}, m_{3}=\frac{n+4 a}{a}$.

If $\operatorname{gcd}(n, m)=1$ then

$$
|m e|_{n}>\frac{n}{2},|m(n-a)|_{n}=n-a>\frac{n}{2},|m(n-b)|_{n}=n-b>\frac{n}{2},
$$

thus $\operatorname{ind}(S)=1$.

Next assume that $\operatorname{gcd}(n, m)>1$. Then $\operatorname{gcd}\left(n, m_{1}\right)=\operatorname{gcd}\left(n, m_{2}\right)=\operatorname{gcd}\left(n, m_{3}\right)=1$. Moreover,

$$
\left|m_{1} e\right|_{n}>\frac{n}{2},\left|m_{2} e\right|_{n}>\frac{n}{2},\left|m_{3} e\right|_{n}>\frac{n}{2},\left|m_{1} a\right|_{n}<\frac{n}{2},\left|m_{2} a\right|_{n}<\frac{n}{2},\left|m_{3} a\right|_{n}<\frac{n}{2} .
$$


If $b<\frac{n}{4}$, we have $\left|m_{1}(n-b)\right|_{n}=n-2 b>\frac{n}{2}$. If $\frac{n}{4}<b<\frac{n}{3}$, we have $\left|m_{3}(n-b)\right|_{n}=2 n-4 b>\frac{n}{2}$. If $\frac{n}{3}<b<\frac{n}{2}$, we have $\left|m_{2}(n-b)\right|_{n}=2 n-3 b>\frac{n}{2}$. Then we can find an integer $m_{i}$ such that $\operatorname{gcd}\left(n, m_{i}\right)=1$ and all of $\left|m_{i} e\right|_{n},\left|m_{i}(n-b)\right|_{n},\left|m_{i}(n-a)\right|_{n}$ are larger than $\frac{n}{2}$, which implies that $\operatorname{ind}(S)=1$.

Renumbering the sequence:

Now we begin to renumber the sequence such that $e<\frac{a}{4}$. For this purpose, by Lemma 2.10, 2.11 and Lemma 2.12, we can assume that $a=p_{2}<2 e$ and $p_{2} \mid c$.

Lemma 2.13. If $a=p_{2}<2 e$ and $a \mid c$, then $\operatorname{ind}(S)=1$ or the sequence $S$ can be renumbered as

$$
\left(e^{\prime} g\right) \cdot\left(c^{\prime} g\right) \cdot\left(\left(n-b^{\prime}\right) g\right) \cdot\left(\left(n-a^{\prime}\right) g\right)
$$

such that $e^{\prime}<a^{\prime} \leq b^{\prime}<c$ and $a \geq 10 e^{\prime}$. Moreover, $e^{\prime}=p_{2}$ or $e^{\prime}=2 p_{2}$.

Proof. Let $m=\frac{n-a}{a}, m_{1}=\frac{n-2 a}{a}, m_{2}=\frac{n+3 a}{2 a}, m_{3}=\frac{n+5 a}{2 a}$.

If $\operatorname{gcd}(n, m)=1$, then $\frac{n}{2}<|m e|_{n}<n-10 a$ and $|m c|_{n}=n-c>\frac{n}{2}$. For this case, if $|m(n-b)|_{n}>\frac{n}{2}$, we have $\operatorname{ind}(S)=1$. Otherwise, it must hold $a<|m(n-b)|_{n}$. We get a renumbering:

$$
e^{\prime}=a, c^{\prime}=|m(n-b)|_{n},\left\{b^{\prime}, a^{\prime}\right\}=\left\{c, n-|m e|_{n}\right\},
$$

and it is easy to check that $a^{\prime} \geq 10 e^{\prime}$.

Next we assume that $\operatorname{gcd}(m, n)>1$, then $p_{2} \mid\left(p_{1} p_{3}-1\right)$ and $\operatorname{gcd}\left(n, m_{1}\right)=\operatorname{gcd}\left(n, m_{2}\right)=$ $\operatorname{gcd}\left(n, m_{3}\right)=1$.

If $c=2 t a$ for some integer $t$. Let $m^{\prime}=\frac{n+a}{2 a}$. Then $\operatorname{gcd}\left(n, m^{\prime}\right)=1$ and $\left|m^{\prime} e\right|_{n}<\frac{n}{2}$, $\left|m^{\prime} c\right|_{n}=\frac{c}{2}<\frac{n}{2},\left|m^{\prime}(n-a)\right|_{n}=\frac{n-a}{2}<\frac{n}{2}$, and $\operatorname{ind}(S)=1$.

If $c=(2 t+1) a$ for some integer $t$. We distinguish three cases according to the value of $\frac{n}{c}$.

Case 1. $\frac{n}{4}>c$. Replace $m$ by $m_{1}$ and repeat the above process, we have $\left|m_{1}(n-b)\right|_{n}>\frac{n}{2}$, $\left|m_{1} c\right|_{n}>\frac{n}{2}$ and $\left|m_{1} e\right|_{n}>\frac{n}{2}$, which implies $\operatorname{ind}(S)=1$, or we can obtain a renumbering:

$$
e^{\prime}=2 a, c^{\prime}=\left|m_{1}(n-b)\right|_{n},\left\{b^{\prime}, a^{\prime}\right\}=\left\{2 c, n-\left|m_{1} e\right|_{n}\right\},
$$

it also holds that $a^{\prime} \geq 10 e^{\prime}$.

Case 2. $\frac{n}{4}<c<\frac{n}{3}$. Then $\left|m_{3}(n-a)\right|_{n}=\frac{n-5 a}{2}<\frac{n}{2}$. We have $\left|m_{3} e\right|_{n}<\frac{n}{2}$ and $\left|m_{3} c\right|_{n}=$ $\left|\frac{n+5 c}{2}\right|_{n}<\frac{n}{2}$, exactly it belongs to $\left(\frac{n}{8}, \frac{n}{3}\right)$. Then $\operatorname{ind}(S)=1$.

Case 3. $\frac{n}{3}<c$. Then $\left|m_{2}(n-a)\right|_{n}=\frac{n-3 a}{2}<\frac{n}{2}$. We have $\left|m_{2} c\right|_{n}=\left|\frac{n+3 c}{2}\right|_{n}<\frac{n}{4},\left|m_{2} e\right|_{n}<\frac{n}{2}$, and hence $\operatorname{ind}(S)=1$.

Through the process of renumbering, we can always assume that $e \in\left\{p_{1}, p_{2}, 2 p_{2}\right\}$ and $a>4 e$. Particularly, $a \geq 10 e$ when $e \in\left\{p_{2}, 2 p_{2}\right\}$. Hence we also assume that $s \leq 9$ by Lemma 2.9.

Let $k_{1}$ be the largest positive integer such that $\left\lceil\frac{\left(k_{1}-1\right) n}{c}\right\rceil=\left\lceil\frac{\left(k_{1}-1\right) n}{b}\right\rceil$ and $\frac{k_{1} n}{c} \leq m<\frac{k_{1} n}{b}$ for some integer $m$. Since $\frac{b n}{c} \leq n-1<n=\frac{b n}{b}$ and $\frac{t n}{b}-\frac{t n}{c}=\frac{t(c-b) n}{b c}>2$ for all $t \geq b$, such integer $k_{1}$ always exists and $k_{1} \leq b$.

As mentioned above, we only need prove Proposition 2.1. We now show that Proposition 2.1 holds through the following 3 propositions.

Proposition 2.14. Suppose $\left\lceil\frac{n}{c}\right\rceil<\left\lceil\frac{n}{b}\right\rceil$, then Proposition 2.1 holds. 
Proposition 2.15. Suppose $\left\lceil\frac{n}{c}\right\rceil=\left\lceil\frac{n}{b}\right\rceil$. Let $k_{1}$ be the largest positive integer such that $\left\lceil\frac{\left(k_{1}-1\right) n}{c}\right\rceil=$ $\left\lceil\frac{\left(k_{1}-1\right) n}{b}\right\rceil$ and $\frac{k_{1} n}{c} \leq m_{1}<\frac{k_{1} n}{b}$ holds for some integer $m_{1}$. If $k_{1}>\frac{b}{a}$, then Proposition 2.1 holds.

Proposition 2.16. Suppose $\left\lceil\frac{n}{c}\right\rceil=\left\lceil\frac{n}{b}\right\rceil$. Let $k_{1}$ be the largest positive integer such that $\left\lceil\frac{\left(k_{1}-1\right) n}{c}\right\rceil=$ $\left\lceil\frac{\left(k_{1}-1\right) n}{b}\right\rceil$ and $\frac{k_{1} n}{c} \leq m_{1}<\frac{k_{1} n}{b}$ holds for some integer $m_{1}$. If $k_{1} \leq \frac{b}{a}$, then Proposition 2.1 holds.

\section{Proof of Proposition 2.14}

In this section, we assume that $\left\lceil\frac{n}{c}\right\rceil<\left\lceil\frac{n}{b}\right\rceil$. Let $m_{1}=\left\lceil\frac{n}{c}\right\rceil$. Then we have $m_{1}-1<\frac{n}{c} \leq$ $m_{1}<\frac{n}{b}$. By Lemma $2.3(1)$, it suffices to find $m$ and $k$ such that $\frac{k n}{c} \leq m<\frac{k n}{b}, \operatorname{gcd}(m, n)=1$, $1 \leq k \leq b$, and $m a<n$. So in what follows, we may always assume that $\operatorname{gcd}\left(n, m_{1}\right)>1$.

Lemma 3.1. If $\left[\frac{n}{c}, \frac{n}{b}\right]$ contains at least two integers, then $\operatorname{ind}(S)=1$.

Proof. Note that $b \geq p_{1} p_{3}$ and $b \geq p_{1} p_{3}$. Thus $\frac{n}{b} \leq p_{2}$ and $\frac{n}{c} \leq p_{1}$. It must hold

$$
m_{1}-1<\frac{n}{c} \leq m_{1}<m_{1}+1<\frac{n}{b}=m_{1}+2=p_{2},
$$

or

$$
m_{1}-1<\frac{n}{c} \leq m_{1}<m_{1}+1 \leq \frac{n}{b}<m_{1}+2 \leq p_{2} .
$$

Clearly, $p_{1}=m_{1}$ or $p_{1}=m_{1}+1$.

If (3.1) holds, we have $m_{1}=p_{1}, 2 p_{3} \mid\left(m_{1}+1\right), m_{1}+2=p_{2}$ and $b=p_{1} p_{3}$. Then we infer that $c=p_{2} p_{3}$ and $a-e=2 p_{3}<2 p_{1} \leq 2 e$, which contradicts to the assumption $a>4 e$.

If (3.2) holds, we have $m_{1} \geq 10$ and

$$
2 m_{1}-2<\frac{2 n}{c} \leq 2 m_{1}<2 m_{1}+1<2 m_{1}+2 \leq \frac{n}{b}<2 m_{1}+4 .
$$

If $\operatorname{gcd}\left(n, 2 m_{1}+1\right)=1$, let $m=2 m_{1}+1$ and $k=2$, we have

$$
m a<\frac{4 m}{3}(a-e)=\frac{4 m}{3}(c-b)<\frac{8 m_{1}+4}{3} \times \frac{3 n}{\left(m_{1}+2\right)\left(m_{1}-1\right)} \leq \frac{7 n}{9}<n,
$$

as desired. If $\operatorname{gcd}\left(n, 2 m_{1}+1\right)>1$, we infer that $p_{2}=2 m_{1}+1$ and $m_{1} \geq 28$. Let $m=3 m_{1}+2$ and $k=3$, we have $\operatorname{gcd}(n, m)=1$ and

$$
m a<\frac{4 m}{3}(a-e)=\frac{4 m}{3}(c-b)<\frac{12 m_{1}+8}{3} \times \frac{3 n}{\left(m_{1}+2\right)\left(m_{1}-1\right)} \leq \frac{172 n}{405}<n,
$$

and $\operatorname{ind}(S)=1$.

By Lemma 3.1, we may assume that $\left[\frac{n}{c}, \frac{n}{b}\right]$ contains exactly one integer $m_{1}$, and thus

$$
m_{1}-1<\frac{n}{c} \leq m_{1}<\frac{n}{b}<m_{1}+1 .
$$

Let $l$ be the smallest integer such that $\left[\frac{l n}{c}, \frac{l n}{b}\right)$ contains at least four integers. Clearly, $l \geq 3$. Since $\frac{n}{b}-m_{1}<1$ and $m_{1}-\frac{n}{c}<1$, by using the minimality of $l$ we obtain that $l m_{1}-4<\frac{l n}{c}<$ $\frac{l n}{b}<l m_{1}+4$. Then $\frac{\ln (c-b)}{b c}=\frac{l n}{b}-\frac{l n}{c}<\left(l m_{1}+4\right)-\left(l m_{1}-4\right)=8$ and thus

$$
l<\frac{8 b c}{(c-b) n}<\frac{8 b}{(a-e)\left(m_{1}-1\right)}<\frac{8 b}{3 e\left(m_{1}-1\right)}<b .
$$


We claim that $\left[\frac{l n}{c}, \frac{l n}{b}\right)$ contains at most six integers. For any positive integer $j$, let $N_{j}$ denote the number of integers contained in $\left[\frac{l n}{c}, \frac{l n}{b}\right)$. Since

$$
\begin{aligned}
& \left(\frac{(j+1) n}{b}-(j+1) m_{1}\right)-\left(\frac{j n}{b}-j m_{1}\right)=\frac{n}{b}-m_{1}<1, \\
& \left((j+1) m_{1}-\frac{(j+1) n}{c}\right)-\left(j m_{1}-\frac{j n}{c}\right)=m_{1}-\frac{n}{c}<1,
\end{aligned}
$$

we infer that $N_{j+1}-N_{j} \leq 2$, it is sufficient to show our claim.

By the claim above we have

$$
l m_{1}-j_{0}<\frac{l n}{c} \leq l m_{1}-j_{0}+1<\cdots<l m_{1}-j_{0}+4<\frac{l n}{b} \leq l m_{1}-j_{0}+6
$$

for some $1 \leq j_{0} \leq 4$. We remark that since $n=p_{1} p_{2} p_{3}$ and $\left[\frac{l n}{c}, \frac{l n}{b}\right)$ contains at least four integers, one of them (say $m$ ) must be co-prime to $n$. If $m a<n$, then we have done by Lemma 2.2(1)(with $k=l<b)$.

Proposition 2.14 can be proved by the following three lemmas.

Lemma 3.2. If $m_{1} \neq 5,7$, then $\operatorname{ind}(S)=1$.

Proof. Since $m_{1} \neq 5,7$, we have $m_{1} \geq 10$ and $n>m_{1} b \geq 10 b$. Let $k=l$ and let $m$ be one of the integers in $\left[\frac{l n}{c}, \frac{l n}{b}\right)$ which is co-prime to $n$. Note that $m \leq l m_{1}+3$ and $l \geq 3$, then

$$
m a \leq\left(l m_{1}+3\right) a<\frac{4\left(l m_{1}+3\right)}{3}\left(\frac{l n}{l m-j_{0}}-\frac{l n}{l m-j_{0}+6}\right)=\frac{4\left(l m_{1}+3\right) \times 6 l n}{3\left(l m_{1}-j_{0}\right)\left(l m_{1}-j_{0}+6\right)}<n .
$$

and we have done.

Lemma 3.3. If $m_{1}=5$, then $\operatorname{ind}(S)=1$.

Proof. Since $m_{1}=5$, we have $4<\frac{n}{c} \leq 5<\frac{n}{b}<6$, thus $a<\frac{4}{3}(c-b)<\frac{n}{9}$. If $\frac{2 n}{c}<9<\frac{2 n}{b}$, let $m=9$ and $k=2$, then we have done. Then $9<\frac{2 n}{c}$, so $a<\frac{4}{3}(c-b)<\frac{2 n}{27}<\frac{n}{13}$.

If $\frac{2 n}{c}<11<\frac{2 n}{b}$, we infer that $\frac{27}{2}<\frac{3 n}{c} \leq 15<16<\frac{33}{2}<\frac{3 n}{b}<18$. If $16 a<n$, let $m=16$ and $k=3$, we have done. If $16 a>a$, then $n<18 a<2 n, 3 n<18 b<18 c<4 n$ and $18 e<\frac{9 a}{2}<n$, let $M=18$. Then $|M e|_{n}+|M c|_{n}+|M(n-b)|_{n}+|M(n-a)|_{n}=M e+(M c-3 n)+(4 n-M b)+(2 n-M a)=$ $3 n$, then we have done.

Next we assume that $9<\frac{2 n}{c} \leq 10<\frac{2 n}{b} \leq 11$.

We infer that $a<\frac{n}{18}$. If $\frac{3 n}{c}<16<\frac{3 n}{b}$, let $m=16$ and $k=3$, then we have done. Otherwise, $\frac{27}{2}<\frac{3 n}{c} \leq 15<\frac{3 n}{b}<16$, hence we obtain $a<\frac{n}{21}$.

If $\frac{27}{2}<\frac{3 n}{c} \leq 14<15<\frac{3 n}{b}<16$, we have $\frac{3 n}{16}<b<\frac{n}{5}<\frac{3 n}{14}<c<\frac{2 n}{9}$. If $24 a<n$, let $M=12$, we have $|M(n-a)|_{n}>\frac{n}{2},|M(n-b)|_{n}>\frac{n}{2},|M c|_{n}>\frac{n}{2}$, then $\operatorname{ind}(S)=1$. If $24 a>n$, we infer that $n<27 a<2 n, 27 e<n$ and $5 n<\frac{81 n}{16}<27 b<27 c<6 n$, let $M=27$. Then $|M e|_{n}+|M c|_{n}+|M(n-b)|_{n}+|M(n-a)|_{n}=M e+(M c-5 n)+(6 n-M b)+(2 n-M a)=3 n$, and we have done.

Next assume that $14<\frac{3 n}{c} \leq 15<\frac{3 n}{b}<16$, we infer that $28 a<n$.

If $\frac{4 n}{c} \leq 19<20<21<\frac{4 n}{b}$, since either 19 or 21 is co-prime to $n$ (otherwise, $n=5 \times 7 \times 19=$ $665<1000$, a contradiction), let $m$ be one of 19,21 such that $\operatorname{gcd}(n, m)=1$ and $k=4$, then we have done. 
If $\frac{4 n}{c} \leq 19<20<\frac{4 n}{b} \leq 21$, let $M=24$, then $4 n<24 b<\frac{24 n}{5}<5 n<\frac{96 n}{19}<24 \times \frac{4 n}{19}<24 c<$ $6 n$, and $|M e|_{n}+|M c|_{n}+|M(n-b)|_{n}+|M(n-a)|_{n}=M e+(M c-5 n)+(5 n-M b)+(n-M a)=n$, and we have done.

If $19<\frac{4 n}{c} \leq 20<21<\frac{4 n}{b}$, if $\operatorname{gcd}(n, 21)=1$, let $m=21$ and $k=4$, then we have done. If $\operatorname{gcd}(n, 21)>1$, then $\operatorname{gcd}(26, n)=1$. Otherwise, $n=5 \times 7 \times 13<1000$, a contradiction. Let $M=26$, we have $4 n<26 b<\frac{104 n}{21}<5 n<\frac{26 n}{5}<26 c<6 n$, and $|M e|_{n}+|M c|_{n}+|M(n-b)|_{n}+|M(n-a)|_{n}=$ $M e+(M c-5 n)+(5 n-M b)+(n-M a)=n$. Then $\operatorname{ind}(S)=1$.

Next assume that $19<\frac{4 n}{c} \leq 20<\frac{4 n}{b} \leq 21$.

If $39<\frac{8 n}{c} \leq 40<\frac{8 n}{b} \leq 41$, we infer that $a<\frac{n}{73}$. Then $b>\frac{n}{6}>\frac{73 a}{6}>12 a$, which contradicts to $s \leq 9$.

If $39<\frac{8 n}{c} \leq 40<41<\frac{8 n}{b} \leq 42$, we infer that $a<\frac{n}{51}$. Let $M=36$, then $|M e|_{n}<9 a<\frac{n}{2}$, $|M c|_{n}<\frac{n}{2}$ and $|M(n-a)|_{n}<\frac{n}{2}$ (otherwise, $72 a<n$ contradicts to $s \leq 9$ ). Exactly, $|M c|_{n}$ belongs to $\left(\frac{n}{5}, \frac{15 n}{39}\right)$. Then $\operatorname{ind}(S)=1$.

If $38<\frac{8 n}{c} \leq 39<40<\frac{8 n}{b} \leq 41$, we infer that $\frac{n}{52}<a<\frac{n}{48}$. If $\operatorname{gcd}(n, 39)=1$, let $m=39$ and $k=8$, then we have done. If $\operatorname{gcd}(n, 39)>1$, then $\operatorname{gcd}(n, 11)=1$, otherwise $n=5 \times 11 \times 13=$ $715<1000$, a contradiction. Let $M=44$, then $\operatorname{gcd}(n, M)=1$ and $|M(n-a)|_{n}<\frac{n}{2}$ (otherwise, $88 a<n$ contradicts to $s \leq 9),|M(n-b)|_{n}<\frac{n}{2},|M c|_{n}<\frac{n}{2}$. Exactly, $|M(n-b)|_{n}$ belongs to $\left(\frac{n}{5}, \frac{13 n}{41}\right)$ and $|M c|_{n}$ belongs to $\left(\frac{n}{39}, \frac{3 n}{19}\right)$. Then $\operatorname{ind}(S)=1$.

Now we infer that $38<\frac{8 n}{c} \leq 39<40<41<\frac{8 n}{b} \leq 42$ and $\frac{n}{53}<a<\frac{n}{37}$. Let $M=36$, we have $|M(n-a)|_{n}<\frac{n}{2}$ (otherwise, $72 a<n$ contradicts to $s \leq 9, M e<9 a<\frac{n}{2}$ and $6 n+\frac{6 n}{7} \leq$ $M b<7 n+\frac{n}{41}$. If $M b<7 n$, then $|M(n-b)|_{n}<\frac{n}{2}$ and $\operatorname{ind}(S)=1$. Hence we infer that $a<\frac{n}{46}$. Moreover, we can assume that $n=5 \times 13 \times 41$. Otherwise, there exists an integer(say $m$ ) between 39 and 41 such that $\operatorname{gcd}(n, m)=1$ and $m a<n$. Let $k=8$, thus ind $(S)=1$. Simply calculating shows that $p_{1}=5, p_{2}=13$. However, we can't find suitable $a$ and $e$ such that $a>4 e$.

Hence we complete the proof.

Lemma 3.4. If $m_{1}=7$, then $\operatorname{ind}(S)=1$.

Proof. Since $m_{1}=7$, we have $6<\frac{n}{c} \leq 7<\frac{n}{b}<8$, thus $a<\frac{4}{3}(c-b)<\frac{n}{18}$.

If $12<\frac{2 n}{c} \leq 13<14<15<\frac{n}{b}<16$, then at least one of $13,14,15$ is co-prime to $n$. Let $m \in[13,15]$ such that $\operatorname{gcd}(n, m)=1$ and $k=1$, then $m a<n$ and $\operatorname{ind}(S)=1$.

If $13<\frac{2 n}{c} \leq 14<15<\frac{2 n}{b}<16$, we have $\frac{n}{8}<b<\frac{2 n}{15}<\frac{n}{7}<c<\frac{2 n}{13}, a<\frac{n}{26}$ and $2 n<16 b<$ $2 n+\frac{2 n}{15}<2 n+\frac{2 n}{7}<16 c<2 n+\frac{6 n}{13}$. If $32 a>n$, let $M=16$, we have $|M e|_{n}<\frac{n}{2},|M c|_{n}<\frac{n}{2}$ and $|M(n-a)|_{n}<\frac{n}{2}$, then $\operatorname{ind}(S)=1$. If $32 a<n$, by inequality $\frac{4 n}{c} \leq 28<29<30<\frac{4 n}{b}$, we infer that $n=5 \times 7 \times 29$. Since $e<\frac{a}{4}<\frac{n}{104}$, we have $e<10$, then $e=p_{1}$. If $p_{1}=7$, then $p_{2}=29$ and $c=5 \times 29, a=29$, thus $b=4 \times 29+7$, which contradicts to $7 \mid b$. We infer that $p_{1}=5$, and thus $\frac{n}{c} \leq \frac{n}{p_{2} p_{3}}=5$, a contradiction.

If $12<\frac{2 n}{c} \leq 13<14<\frac{2 n}{b}<15$, we infer that $a<\frac{n}{22}$ and $91 \mid n$. we also assume that $27 a>n$. Otherwise, let $m=27$ and $k=4$, we have $\frac{4 n}{c} \leq 26<27<28<\frac{42 n}{b}$. If $5 \mid n$, then $n=5 \times 91=455<1000$, a contradiction. Thus $\operatorname{gcd}(n, 30)=1$. Let $M=30$, we have $M e<8 a<n$, $4 n<30 b<30 c<5 n$ and $n<30 a<2 n$. Then $|M e|_{n}+|M c|_{n}+|M(n-b)|_{n}+|M(n-a)|_{n}=$ $M e+(M c-4 n)+(5 n-M b)+(2 n-M a)=3 n$, and $\operatorname{ind}(S)=1$. 
Next assume that $13<\frac{2 n}{c} \leq 14<\frac{2 n}{b}<15$, and we infer that $a<\frac{n}{36}$.

If $\frac{4 n}{c}<27<\frac{4 n}{b}$, let $m=27$ and $k=4$, then we have done. So $27<\frac{4 n}{c} \leq 28<\frac{4 n}{b}<30$, and $a<\frac{n}{50}$.

If $27<\frac{4 n}{c} \leq 28<29<\frac{4 n}{b}<30$, we have $\frac{2 n}{15}<b<\frac{4 n}{29}<\frac{n}{7} \leq c<\frac{4 n}{27}$ and $\frac{24 n}{5}<b<\frac{144 n}{29}<$ $5 n<\frac{36 n}{7} \leq c<\frac{48 n}{9}<6 n$. Let $M=36$, we have $|M e|_{n}+|M c|_{n}+|M(n-b)|_{n}+|M(n-a)|_{n}=$ $M e+(M c-5 n)+(5 n-M b)+(n-M a)=n$, and $\operatorname{ind}(S)=1$.

If $27<\frac{4 n}{c} \leq 28<\frac{4 n}{b} \leq 29$, we infer that $a<\frac{n}{73}$, then $b>\frac{4 n}{29}>\frac{292 a}{29}>10 a$, which contradicts to $s \leq 9$.

We complete the proof.

\section{Proof of Proposition 2.15}

In this section, we always assume that $\left\lceil\frac{n}{c}\right\rceil=\left\lceil\frac{n}{b}\right\rceil$, so $k_{1} \geq 2$, and we also assume that $k_{1}>\frac{b}{a}$. Proposition 2.15 can be proved through the following two lemmas.

Lemma 4.1. If the assumption is as in Proposition 2.15, then $k_{1}<3$.

Proof. If $k_{1} \geq 3$, then $\frac{\left(k_{1}-1\right) n}{b}-\frac{\left(k_{1}-1\right) n}{c}=\frac{(a-e)\left(k_{1}-1\right) n}{b c} \geq \frac{3 a}{4} \frac{2 k_{1} n}{3 b c}>1$, a contradiction.

Lemma 4.2. If the assumption is as in Proposition 2.15 and $k_{1}=2$, then $\operatorname{ind}(S)=1$.

Proof. If $\frac{n}{c}>3$, then $\frac{n}{b}-\frac{n}{c}=\frac{(a-e) n}{b c} \geq \frac{2 a}{3} \frac{n}{b c}>1$, a contradiction.

If $\frac{n}{c} \leq 3<\frac{n}{b}$, we have $n<3 c<2 n, 3 a<3 b<n$. Let $m=3$, then $\operatorname{gcd}(n, m)=1$ and $|m e|_{n}+|m c|_{n}+|m(n-b)|_{n}+|m(n-a)|_{n}=m e+(m c-n)+(n-m b)+(n-m a)=n$, we have done.

If $\frac{n}{c}<\frac{n}{b}<3$, then $\frac{n}{3}<b<2 a$, and $2 n<6 c<3 n, 2 n<6 b<3 n, 6 a>3 b>n$. $6 e<2 a<n$. Let $m=6$, then $\operatorname{gcd}(n, m)=1$, and $3 n \geq|m e|_{n}+|m c|_{n}+|m(n-b)|_{n}+|m(n-a)|_{n} \geq$ $m e+(m c-2 n)+(3 n-m b)+(2 n-m a)=3 n$, we have done.

\section{Proof of Proposition 2.16}

In this section, we always assume that $\left\lceil\frac{n}{c}\right\rceil=\left\lceil\frac{n}{b}\right\rceil$, so $k_{1} \geq 2$, and we also assume that $k_{1}<\frac{b}{a}$, hence $s \geq k_{1}$. Proposition 2.16 can be proved by the following Lemmas 5.1-5.6 and Lemma 5.9.

Lemma 5.1. If the assumption is as in Proposition 2.16, then $k_{1} \leq 7$.

Proof. If $k_{1} \geq 8$, then $\frac{\left(k_{1}-1\right) n}{b}-\frac{\left(k_{1}-1\right) n}{c} \geq \frac{(a-e) 7 n}{b c} \geq \frac{21 a n}{4 b c}>\frac{21}{20}>1$, a contradiction.

Lemma 5.2. If the assumption is as in Proposition 2.16 and $k_{1}=7$, then $\operatorname{ind}(S)=1$.

Proof. If $c \leq \frac{9 n}{20}$, we have $\frac{\left(k_{1}-1\right) n}{b}-\frac{\left(k_{1}-1\right) n}{c} \geq \frac{(a-e) 6 n}{b c} \geq \frac{9 a n}{2 b c} \geq \frac{10 a}{b}>1$, a contradiction. Thus $\frac{n}{c}<\frac{20}{9}$ and we infer that $12<\frac{6 n}{c}<\frac{6 n}{b} \leq 13$ or $13<\frac{6 n}{c}<\frac{6 n}{b} \leq 14$. 
Case 1. It holds that $12<\frac{6 n}{c}<\frac{6 n}{b} \leq 13$. Then $a<\frac{n}{19}$ and we have

$$
\begin{gathered}
14<\frac{7 n}{c} \leq 15<\frac{7 n}{b} \leq \frac{91}{6} \\
16<\frac{8 n}{c} \leq 17<\frac{8 n}{b} \leq \frac{52}{3} \\
18<\frac{9 n}{c} \leq 19<\frac{9 n}{b} \leq \frac{39}{2} \\
20<\frac{10 n}{c} \leq 21<\frac{10 n}{b} \leq \frac{65}{3} .
\end{gathered}
$$

If $\operatorname{gcd}(n, 15)=1$, let $m=15$ and $k=7$, then we have done. If $\operatorname{gcd}(n, 17)=1$, let $m=17$ and $k=8$, then we have done. If $\operatorname{gcd}(n, 19)=1$, let $m=19$ and $k=9$, then we have done.

Now assume that $n=5 \times 17 \times 19$ and thus $\operatorname{gcd}(n, 21)=1$. If $21 a<n$, let $m=21$ and $k=10$, then we have done. If $21 a>n$, let $M=12$, we have $|M(n-a)|_{n}<\frac{n}{2}$ and $|M e|_{n}<\frac{n}{2}$. Moreover, $5 n+\frac{7 n}{13}=\frac{72 n}{13}<12 b<\frac{28 n}{5}=5 n+\frac{3 n}{5}$, which implies that $|M(n-b)|_{n}<\frac{n}{2}$. So $\operatorname{ind}(S)=1$.

Case 2. It holds that $13<\frac{6 n}{c}<\frac{6 n}{b} \leq 14$. Then $a<\frac{n}{22}$ and $\frac{91}{6}<\frac{7 n}{c}<16<\frac{7 n}{b} \leq \frac{49}{3}$. Let $m=16$ and $k=7$, we have $\operatorname{gcd}(n, m)=1$ and $m a<n$, then $\operatorname{ind}(S)=1$.

Lemma 5.3. If the assumption is as in Proposition 2.16 and $k_{1}=6$, then $\operatorname{ind}(S)=1$.

Proof. Similar to Lemma 5.2, we have $\frac{n}{c}<\frac{8}{3}$ and we infer that $10<\frac{5 n}{c}<\frac{5 n}{b} \leq 11$, or $11<\frac{5 n}{c}<$ $\frac{5 n}{b} \leq 12$, or $12<\frac{5 n}{c}<\frac{5 n}{b} \leq 13$, or $13<\frac{5 n}{c}<\frac{5 n}{b} \leq \frac{40}{3}$.

Case 1. It holds that $10<\frac{5 n}{c}<\frac{5 n}{b} \leq 11$, then $a<\frac{n}{16}$. If $18 a>n$. Let $M=9$, we infer that $|M(n-a)|_{n}<\frac{n}{2},|M e|_{n}<\frac{n}{2}$ and $|M c|_{n}<\frac{n}{2}$ (exactly, $\left.4 n+\frac{n}{11}<9 c<4 n+\frac{n}{2}\right)$. Then $\operatorname{ind}(S)=1$. Moreover, we have

$$
\frac{6 n}{c} \leq 13<\frac{6 n}{b}, \quad \frac{7 n}{c} \leq 15<\frac{7 n}{b}, \quad \frac{8 n}{c} \leq 17<\frac{8 n}{b} .
$$

If $\operatorname{gcd}(n, 13)=1$, let $m=13$ and $k=6$, if $\operatorname{gcd}(n, 15)=1$, let $m=15$ and $k=7$, if $\operatorname{gcd}(n, 17)=1$, let $m=17$ and $k=8$, then $m a<n$ and $\operatorname{ind}(S)=1$. If none of the three integers is co-prime to $n$, then $n=5 \times 13 \times 17$ and $p_{1}=13, p_{2}=17, p_{3}=5$.

By the renumbering process, we may assume that $17 \leq e \leq \frac{a}{10}$ or $a \geq 4 \times 17$. If $17 \leq e \leq \frac{a}{10}$, then $e \leq \frac{n}{180}<10$, a contradiction. If $a \geq 4 \times 17$, then $a \geq \frac{4 n}{5 \times 13}>\frac{n}{17}$, a contradiction.

Case 2. It holds that $11<\frac{5 n}{c}<\frac{5 n}{b} \leq 12$. Then $a<\frac{4}{3}(a-e)=\frac{4}{3}(c-b)<\frac{4}{3}\left(\frac{5 n}{11}-\frac{n}{12}\right)<\frac{n}{19}$. If $\frac{8 n}{c}<18<\frac{8 n}{b}$, let $m=18$ and $k=8$, then we have done. Otherwise, it holds $18<\frac{8 n}{c} \leq 19<$ $\frac{8 n}{b} \leq \frac{96}{5}$, and thus $a<\frac{4}{3}\left(\frac{4 n}{9}-\frac{5}{12}\right)=\frac{n}{27}$. We infer that $\frac{b}{a}>\frac{5 n}{12} \times \frac{27}{n}>10$, which contradicts to $s \leq 9$.

Case 3. It holds that $12<\frac{5 n}{c}<\frac{5 n}{b} \leq 13$. Then $a<\frac{4}{3}(a-e)=\frac{4}{3}(c-b)<\frac{4}{3}\left(\frac{5 n}{12}-\frac{n}{13}\right)<\frac{n}{23}$. If $\frac{7 n}{c}<18<\frac{7 n}{b}$, let $m=18$ and $k=7$, then we have done. Otherwise, it holds $\frac{84}{5}<\frac{7 n}{c} \leq 17<$ $\frac{7 n}{b} \leq 18$, and thus $a<\frac{4}{3}\left(\frac{5 n}{12}-\frac{7}{18}\right)=\frac{n}{27}$. We infer that $\frac{b}{a}>\frac{7 n}{18} \times \frac{27}{n}>10$, which contradicts to $s \leq 9$.

Case 4. It holds that $13<\frac{5 n}{c}<\frac{5 n}{b} \leq \frac{40}{3}$. Then $a<\frac{4}{3}(a-e)=\frac{4}{3}(c-b)<\frac{4}{3}\left(\frac{5 n}{13}-\frac{3 n}{8}\right)=\frac{n}{78}$. Since $s \geq k_{1}=6$, by Lemma 2.7, we have $b>\frac{n}{4}$, thus $\frac{b}{a}>\frac{78}{4}>19$, which contradicts to $s \leq 9$.

Lemma 5.4. If the assumption is as in Proposition 2.16 and $k_{1}=5$, then $\operatorname{ind}(S)=1$.

Proof. Similar to Lemma 5.2, we have $\frac{n}{c}<\frac{10}{3}$, then $8+t<\frac{4 n}{c}<\frac{4 n}{b} \leq 9+t$ for some $t \in[0,4]$ or $13<\frac{4 n}{c}<\frac{4 n}{b} \leq \frac{40}{3}$. We distinguish six cases. 
Case 1. It holds that $13<\frac{4 n}{c}<\frac{4 n}{b} \leq \frac{40}{3}$. We have $39<\frac{12 n}{c}<\frac{12 n}{b} \leq 40$, which contradicts to the maximality of $k_{1}$.

Case 2. $t=0$.

It holds that $8<\frac{4 n}{c}<\frac{4 n}{b} \leq 9$, and we infer that $a<\frac{n}{13}$. Moreover, we have

$$
\frac{5 n}{c} \leq 11<\frac{5 n}{b}, \quad \frac{6 n}{c} \leq 13<\frac{6 n}{b}, \quad \frac{7 n}{c} \leq 15<\frac{7 n}{b}<16 .
$$

If $16 a>n$, let $M=16$, then $M e<n, n<M a<2 n$ and $7 n<M b<M c<8 n$. We infer that $|M e|_{n}+|M c|_{n}+|M(n-b)|_{n}+|M(n-a)|_{n}=3 n$, and $\operatorname{ind}(S)=1$. Thus $16 a<n$. If $\operatorname{gcd}(n, 11)=1$, let $m=11$ and $k=5$, if $\operatorname{gcd}(n, 13)=1$, let $m=13$ and $k=6$, if $\operatorname{gcd}(n, 15)=1$, let $m=15$ and $k=7$, then $m a<n$ and $\operatorname{ind}(S)=1$. If none of $11,13,15$ is co-prime to $n$, then $n=5 \times 11 \times 13=715<1000$, a contradiction.

Case 3. $t=1$.

It holds that $9<\frac{4 n}{c}<\frac{4 n}{b} \leq 10$. We infer that $a<\frac{n}{16}$ and $\frac{45}{4}<\frac{5 n}{c}<12<\frac{5 n}{b} \leq \frac{25}{2}$. Let $m=12$ and $k=5$, then we have done.

Case 4. $t=2$.

It holds that $10<\frac{4 n}{c}<\frac{4 n}{b} \leq 11$. We infer that $a<\frac{n}{20}$ and $15<\frac{6 n}{c}<16<\frac{6 n}{b} \leq \frac{33}{2}$. Let $m=16$ and $k=6$, then we have done.

Case 5. $t=3$.

It holds that $11<\frac{4 n}{c}<\frac{4 n}{b} \leq 12$, and we infer that $a<\frac{n}{24}$. Moreover, we have

$$
\frac{5 n}{c} \leq 14<\frac{5 n}{b} \leq 15, \quad \frac{6 n}{c} \leq 17<\frac{6 n}{b} \leq 18, \quad \frac{7 n}{c} \leq 20<\frac{7 n}{b} \leq 21 .
$$

If $\operatorname{gcd}(n, 14)=1$, let $m=14$ and $k=5$, if $\operatorname{gcd}(n, 17)=1$, let $m=17$ and $k=6$, if $\operatorname{gcd}(n, 20)=1$, let $m=20$ and $k=7$, then $m a<n$ and $\operatorname{ind}(S)=1$. If none of the three integers is co-prime to $n$, then $n=5 \times 7 \times 17=595<1000$, a contradiction.

Case 6. $t=4$.

It holds that $12<\frac{4 n}{c}<\frac{4 n}{b} \leq 13$, then $a<\frac{4}{3}\left(\frac{n}{12}-\frac{n}{13}\right)<\frac{n}{29}$ and $15<\frac{5 n}{c}<16<\frac{5 n}{b} \leq \frac{65}{4}$. Let $m=16$ and $k=5$, we have $\operatorname{ind}(S)=1$.

Lemma 5.5. If the assumption is as in Proposition 2.16 and $k_{1}=4$, then $\operatorname{ind}(S)=1$.

Proof. Similar to Lemma 5.2, we have $\frac{n}{c}<\frac{40}{9}$, then $6+t<\frac{3 n}{c}<\frac{3 n}{b} \leq 7+t$ for some $t \in[0,6]$ or $13<\frac{3 n}{c}<\frac{3 n}{b} \leq \frac{40}{3}$. We distinguish eight cases.

Case 1. It holds that $13<\frac{3 n}{c}<\frac{3 n}{b} \leq \frac{40}{3}$. We have $39<\frac{9 n}{c}<\frac{9 n}{b} \leq 40$, which contradicts to the maximality of $k_{1}$.

Case 2. $t=0$.

It holds that $6<\frac{3 n}{c}<\frac{3 n}{b} \leq 7$. We infer that $a<\frac{n}{10}$ and $8<\frac{4 n}{c}<9<\frac{4 n}{b} \leq \frac{28}{3}$. Let $m=9$ and $k=4$, we have $\operatorname{ind}(S)=1$.

Case 3. $t=1$.

It holds that $7<\frac{3 n}{c}<\frac{3 n}{b} \leq 8$, and we infer that $a<\frac{n}{13}$. If $\frac{5 n}{c}<12<\frac{5 n}{b}$, let $m=12$ and $k=5$, then $\operatorname{ind}(S)=1$. We may assume that $12<\frac{5 n}{c} \leq 13<\frac{5 n}{b}<\frac{40}{3}$, then $a<\frac{n}{18}$. Let $M=18$, 
we have

$$
8 n>\frac{15 n}{2}>18 c>\frac{36 n}{5}>7 n>\frac{90 n}{13}>18 b>\frac{27 n}{4}>6 n,
$$

and $|M e|_{n}+|M c|_{n}+|M(n-b)|_{n}+|M(n-a)|_{n}=M e+(M c-7 n)+(7 n-M b)+(n-M a)=n$, then $\operatorname{ind}(S)=1$.

Case 4. $t=2$.

It holds that $8<\frac{3 n}{c}<\frac{3 n}{b} \leq 9$, and we infer that $a<\frac{n}{18}$. Since

$$
\frac{4 n}{c} \leq 11<\frac{4 n}{b}, \frac{5 n}{c} \leq 14<\frac{5 n}{b}, \frac{6 n}{c} \leq 17<\frac{6 n}{b},
$$

we infer that $n=7 \times 11 \times 17$.

If $18<\frac{56}{3}<\frac{7 n}{c}<19<\frac{7 n}{b} \leq 20$, we have $a<\frac{n}{30}$, let $m=19$ and $k=7$. Then $\operatorname{gcd}(n, m)=1$ and $\operatorname{ind}(S)=1$.

If $19<\frac{7 n}{c}<20<\frac{7 n}{b} \leq 21$, we have $a<\frac{n}{21}$, let $m=20$ and $k=7$. Then $\operatorname{gcd}(n, m)=1$ and $\operatorname{ind}(S)=1$.

If $\frac{56}{3}<\frac{7 n}{c}<19<20<\frac{7 n}{b} \leq 21$, we infer that $19 a>n$. If $27 c<10 n$, then $a<\frac{4}{3}(c-b)<$ $\frac{4}{3}\left(\frac{10 n}{27}-\frac{n}{3}\right)<\frac{n}{20}$, a contradiction. So $\frac{3 n}{8}>c>\frac{10 n}{27}>\frac{7 n}{20}>b>\frac{n}{3}$ and $\frac{81 n}{8}>27 c>10 n>\frac{189 n}{20}>$ $27 b>9 n, n<27 a<\frac{3 n}{2}$. Let $M=27$, we have $|M c|_{n}>\frac{n}{2},|M(n-b)|_{n}>\frac{n}{2},|M(n-a)|_{n}>\frac{n}{2}$, and $\operatorname{ind}(S)=1$.

Case 5. $t=3$.

It holds that $9<\frac{3 n}{c}<\frac{3 n}{b} \leq 10$, and we infer that $a<\frac{n}{22}$ and $\frac{5 n}{c}<16<\frac{5 n}{b}$. Let $m=16$ and $k=5$, then $\operatorname{ind}(S)=1$.

Case 6. $t=4$.

It holds that $10<\frac{3 n}{c}<\frac{3 n}{b} \leq 11$, and we infer that $a<\frac{n}{27}$. If $\frac{5 n}{c}<18<\frac{5 n}{b}$, let $m=18$ and $k=5$, then we have done. If $\frac{7 n}{c}<24<\frac{7 n}{b}$, let $m=24$ and $k=7$, then we have done. Otherwise, we have $\frac{5 n}{18}<b<c<\frac{7 n}{24}$ and $a<\frac{4}{3}(c-b)<\frac{n}{54}$. Then $b>\frac{5 n}{18}>\frac{5 \times 54 a}{18}=15 a$, which contradicts to $s \leq 9$.

Case 7. $t=5$.

It holds that $11<\frac{3 n}{c}<\frac{3 n}{b} \leq 12$, and we infer that $a<\frac{n}{33}$ and

$$
\frac{4 n}{c} \leq 15<\frac{5 n}{b}, \frac{5 n}{c} \leq 19<\frac{5 n}{b}, \frac{6 n}{c} \leq 23<\frac{6 n}{b} .
$$

If $\operatorname{gcd}(n, 17)=1$, let $m=17$ and $k=4$, if $\operatorname{gcd}(n, 21)=1$, let $m=21$ and $k=5$, if $\operatorname{gcd}(n, 25)=1$, let $m=25$ and $k=6$, then $m a<n$ and $\operatorname{ind}(S)=1$. If none of the three integers is co-prime to $n$, then there exists an integer $m \in[26,27]$ belongs to $\left[\frac{7 n}{c}, \frac{7 n}{b}\right)$, let $k=7$, then $\operatorname{gcd}(m, n)=1$ and $\operatorname{ind}(S)=1$.

Case 8. $t=6$.

It holds that $12<\frac{3 n}{c}<\frac{3 n}{b} \leq 13$, then we infer that $a<\frac{n}{39}$ and

$$
\frac{4 n}{c} \leq 17<\frac{5 n}{b}, \frac{5 n}{c} \leq 21<\frac{5 n}{b}, \frac{6 n}{c} \leq 25<\frac{6 n}{b} .
$$

If $\operatorname{gcd}(n, 17)=1$, let $m=17$ and $k=4$, if $\operatorname{gcd}(n, 21)=1$, let $m=21$ and $k=5$, if $\operatorname{gcd}(n, 25)=1$, let $m=25$ and $k=6$, then $m a<n$ and $\operatorname{ind}(S)=1$. If none of the three integers is co-prime to $n$, then $n=5 \times 7 \times 17=595<1000$, a contradiction. 
Lemma 5.6. If the assumption is as in Proposition 2.16 and $k_{1}=3$, then $\operatorname{ind}(S)=1$.

Proof. Similar to Lemma 5.2, we have $\frac{n}{c}<\frac{20}{3}$, then $4+t<\frac{2 n}{c}<\frac{2 n}{b} \leq 5+t$ for some $t \in[0,8]$ or $13<\frac{2 n}{c}<\frac{2 n}{b} \leq \frac{40}{3}$. We distinguish ten cases.

Case 1. It holds that $13<\frac{2 n}{c}<\frac{2 n}{b} \leq \frac{40}{3}$. We have $39<\frac{6 n}{c}<\frac{6 n}{b} \leq 40$, which contradicts to the maximality of $k_{1}$.

Case 2. $t=0$.

It holds that $4<\frac{2 n}{c}<\frac{2 n}{b} \leq 5$. We infer that $7 a<n, 6<\frac{3 n}{c} \leq 7<\frac{3 n}{b} \leq \frac{15}{2}$, and $8<\frac{4 n}{c}<9<\frac{4 n}{b} \leq 10$. If $9 a<n$, let $m=9$ and $k=4$, then we have done. If $9 a>n$, let $M=18$, then $7 n<\frac{36 n}{5}<18 b<\frac{54 n}{7}<8 n<18 c<9 n$, and $18 e<5 a<n$. Then $|M e|_{n}+|M c|_{n}+|M(n-b)|_{n}+|M(n-a)|_{n}=3 n$, and $\operatorname{ind}(S)=1$.

Case 3. $t=1$.

It holds that $5<\frac{2 n}{c}<\frac{2 n}{b} \leq 6$. We infer that $11 a<n$ and $\frac{3 n}{c}<8<\frac{3 n}{b}$. Let $m=8$ and $k=3$, then $\operatorname{ind}(S)=1$.

Case 4. $t=2$.

It holds that $6<\frac{2 n}{c}<\frac{2 n}{b} \leq 7$. We infer that $15 a<n$ and

$$
\frac{3 n}{c}<10<\frac{3 n}{b}, \frac{4 n}{c}<13<\frac{4 n}{b},
$$

thus $\operatorname{gcd}(n, 10)>1, \operatorname{gcd}(n, 13)>1$.

Subcase 4.1. $\frac{5 n}{c}<16<\frac{5 n}{b} \leq \frac{35}{2}$. If $16 a<n$, let $m=16$ and $k=5$, then we have done. If $16 a>n$, let $M=18$, we have $5 n<18 b<18 c<6 n$ and $|M e|_{n}+|M c|_{n}+|M(n-b)|_{n}+|M(n-a)|_{n}=$ 3n. Then $\operatorname{ind}(S)=1$.

Subcase 4.2. $16<\frac{5 n}{c} \leq 17<\frac{5 n}{b} \leq \frac{35}{2}$. We infer that $28 a<n$. Then $\operatorname{gcd}(n, 17)>1$ and $n=5 \times 13 \times 17$. Let $M=27$, we have

$$
9 n>\frac{135 n}{16}>27 c>\frac{81 n}{10}>8 n=\frac{136 n}{17}>\frac{135 n}{17}>27 b>\frac{54 n}{7}>7 n,
$$

then $|M e|_{n}+|M c|_{n}+|M(n-b)|_{n}+|M(n-a)|_{n}=3 n$ and $\operatorname{ind}(S)=1$.

Case 5. $t=3$.

It holds that $7<\frac{2 n}{c}<\frac{2 n}{b} \leq 8$. We infer that $21 a<n$ and

$$
\frac{3 n}{c}<11<\frac{3 n}{b}, \frac{4 n}{c}<15<\frac{4 n}{b},
$$

thus $\operatorname{gcd}(n, 11)>1, \operatorname{gcd}(n, 15)>1$.

Subcase 5.1. $\frac{5 n}{c}<18<\frac{5 n}{b} \leq 20$. Let $m=18$ and $k=5$, then we have done.

Subcase 5.2. $18<\frac{5 n}{c} \leq 19<\frac{5 n}{b} \leq 20$. We infer that $27 a<n$ and thus $\operatorname{gcd}(n, 19)>1$. In further, $\frac{7 n}{c} \leq \frac{77}{3}<26<\frac{133}{5}<\frac{7 n}{b}$, let $m=26$ and $k=7$, then $\operatorname{gcd}(n, 26)=1$ and $\operatorname{ind}(S)=1$.

Case 6. $t=4$.

It holds that $8<\frac{2 n}{c}<\frac{2 n}{b} \leq 9$. We infer that $27 a<n$ and

$$
\frac{3 n}{c}<13<\frac{3 n}{b}, \frac{4 n}{c}<17<\frac{4 n}{b},
$$

thus $\operatorname{gcd}(n, 13)>1, \operatorname{gcd}(n, 17)>1$. 
Subcase 6.1. $\frac{5 n}{c} \leq 21<22<\frac{5 n}{b}$. Let $k=5$ and $m \in[21,22]$ such that $\operatorname{gcd}(n, m)=1$, then we have done.

Subcase 6.2. $21<\frac{5 n}{c} \leq 22<\frac{5 n}{b} \leq \frac{45}{2}$. We infer that $a<\frac{4 n}{189}$ and thus $\frac{b}{a}>\frac{2}{9} \times \frac{189}{4}=\frac{21}{2}>10$, which contradicts to $s \leq 9$.

Subcase 6.3. $20<\frac{5 n}{c} \leq 21<\frac{5 n}{b} \leq 22$. We infer that $33 a<n, 7 \mid n$ and thus $\operatorname{gcd}(n, 33)=1$. Let $M=33$, we have $\frac{15 n}{2}<33 b<\frac{99 n}{13}, \frac{n}{2}<33 a<n$ (otherwise, $66 a<n<\frac{22 b}{5}$, thus $b>15 a$, a contradiction) and $33 e<9 a<n$. Then $|M e|_{n}<\frac{n}{2},|M(n-b)|_{n}<\frac{n}{2},|M(n-a)|_{n}<\frac{n}{2}$, and $\operatorname{ind}(S)=1$.

Case 7. $t=5$.

It holds that $9<\frac{2 n}{c}<\frac{2 n}{b} \leq 10$. We infer that $33 a<n$. If $\frac{5 n}{c}<24<\frac{5 n}{b}$. Let $k=5$ and $m=24$, then $\operatorname{gcd}(n, m)=1$ and $\operatorname{ind}(S)=1$. Otherwise, assume that $\frac{45}{2}<\frac{5 n}{c} \leq 23<\frac{5 n}{b}<24$. We infer that $54 a<n$ and $\frac{b}{a}>\frac{5}{24} \times 54=\frac{90}{8}>10$, which contradicts to $s \leq 9$.

Case 8. $t=6$.

It holds that $10<\frac{2 n}{c}<\frac{2 n}{b} \leq 11$. We infer that $41 a<n$ and $\frac{3 n}{c}<16<\frac{3 n}{b}$. Let $k=3$ and $m=16$, then $\operatorname{gcd}(n, m)=1$ and $\operatorname{ind}(S)=1$.

Case 9. $t=7$.

It holds that $11<\frac{2 n}{c}<\frac{2 n}{b} \leq 12$. We infer that $49 a<n$ and

$$
\frac{3 n}{c}<17<\frac{3 n}{b}, \frac{4 n}{c}<23<\frac{4 n}{b},
$$

thus $\operatorname{gcd}(n, 17)>1, \operatorname{gcd}(n, 23)>1$.

Subcase 9.1. $\frac{5 n}{c} \leq 28<29<\frac{5 n}{b}$. Let $k=5$ and $m \in[28,29]$ such that $\operatorname{gcd}(n, m)=1$, then we have done.

Subcase 9.2. $28<\frac{5 n}{c} \leq 29<\frac{5 n}{b} \leq 30$. We infer that $a<\frac{n}{63}$ and thus $\frac{b}{a}>\frac{21}{2}>10$, which contradicts to $s \leq 9$.

Subcase 9.3. $\frac{55}{2}<\frac{5 n}{c} \leq 28<\frac{5 n}{b} \leq 29$. Similar to Subcase 9.2., we get a contradiction.

Case 10. $t=8$.

It holds that $12<\frac{2 n}{c}<\frac{2 n}{b} \leq 13$, we infer that $a<\frac{n}{58}$. If $\frac{5 n}{c}<32<\frac{5 n}{b}$, let $m=32$ and $k=5$, then we have done. Otherwise, we have $30<\frac{5 n}{c}<\frac{5 n}{b}<32$ and $a<\frac{4}{3}(a-e)=\frac{4}{3}(c-b)<$ $\frac{4}{3}\left(\frac{n}{6}-\frac{5 n}{32}\right)=\frac{n}{72}$. Then $b>\frac{5 n}{32}>\frac{5 \times 72 a}{32}=\frac{45 a}{4}>10 a$, which contradicts to $s \leq 9$.

Lemma 5.7. Suppose that $p_{3}=5, p_{1} \geq 13$ and $6 a<n$. If $e=2 p_{2}$, then $p_{1} \geq 23$ and $n>57 e$. If $a \leq p_{1}$, then $n>85 e$.

Proof. This result can be checked directly.

Lemma 5.8. Let the assumption be as in Proposition 2.16. If $k_{1}=2,4<\frac{2 n}{c} \leq 5<\frac{2 n}{b} \leq 6$ and $5 \mid n$, then $\operatorname{ind}(S)=1$.

Proof. If $n<6 a, m e<2 a<n, 2 n<6 b<6 c<3 n$, let $M=6$. Then $|M e|_{n}+|M c|_{n}+\mid M(n-$ $b)\left.\right|_{n}+|M(n-a)|_{n} \geq M e+(M c-2 n)+(3 n-M b)+(2 n-M a)=3 n$, and $\operatorname{ind}(S)=1$. Next we assume that $6 a<n$ and distinguish three cases.

Case 1. $7<\frac{3 n}{c} \leq 8<\frac{3 n}{b} \leq 9$. 
If $8 a<n$, let $m=8$ and $k=3$, we have ind $(S)=1$. If $8 a>n$, let $M=9$, we have $3 n<9 b<9 c<4 n, 9 e<3 a<n$ and $9 a<2 n$, so $|M e|_{n}+|M c|_{n}+|M(n-b)|_{n}+|M(n-a)|_{n}=$ $M e+(M c-3 n)+(4 n-M b)+(2 n-M a)=3 n$. Then $\operatorname{ind}(S)=1$.

Case 2. $6<\frac{3 n}{c} \leq 7<\frac{3 n}{b} \leq 8$.

If $8 a>n$, let $M=8$, we have $3 n<8 b<8 c<4 n, 8 e<2 a<n$ and $8 a<2 n$, so $|M e|_{n}+|M c|_{n}+|M(n-b)|_{n}+|M(n-a)|_{n}=M e+(M c-3 n)+(4 n-M b)+(2 n-M a)=3 n$. Then $\operatorname{ind}(S)=1$.

Next assume that $8 a<n$, then $7 \mid n, \operatorname{gcd}(n, 11)=\operatorname{gcd}(n, 13)=1$ and $\frac{11 n}{2}>11 c>\frac{33 n}{7}>\frac{22 n}{5}>$ $11 b>\frac{33 n}{8}$.

If $11 c<5 n$, let $M=12$, we have $\frac{60 n}{11}>12 c>\frac{36 n}{7}>\frac{24 n}{5}>12 b>\frac{9 n}{2}$ and $12 e<3 a<\frac{n}{2}$. Then $|M e|_{n}<\frac{n}{2},|M c|_{n}<\frac{n}{2},|M(n-b)|_{n}<\frac{n}{2}$, and $\operatorname{ind}(S)=1$.

If $11 c>5 n$ and $11 a<n$, let $M=11$, then $|M e|_{n}+|M c|_{n}+|M(n-b)|_{n}+|M(n-a)|_{n}=$ $M e+(M c-5 n)+(5 n-M b)+(n-M a)=n$, and $\operatorname{ind}(S)=1$.

Let $11 c>5 n$ and $11 a>n$. If $18 b>7 n$, let $M=9$, we have $\frac{9 n}{2}>9 c>\frac{45 n}{11}>\frac{18 n}{5}>9 b>\frac{7 n}{2}$, then $|M e|_{n}<\frac{n}{2},|M c|_{n}<\frac{n}{2},|M(n-b)|_{n}<\frac{n}{2}$, and $\operatorname{ind}(S)=1$. If $18 b<7 n$, let $M=18$, we have $|M e|_{n}+|M c|_{n}+|M(n-b)|_{n}+|M(n-a)|_{n}=M e+(M c-8 n)+(7 n-M b)+(2 n-M a)=n$, and $\operatorname{ind}(S)=1$.

If $11 c>5 n$ and $11 a>n$, let $M=9$, then $\frac{65 n}{11}>13 c>\frac{39 n}{7}>\frac{26 n}{5}>9 b>\frac{39 n}{8}$. We infer that $|M c|_{n}<\frac{n}{2},|M(n-b)|_{n}+|M(n-a)|_{n}=M e+(M c-4 n)+(5 n-M b)+(2 n-M a)=3 n$, and $\operatorname{ind}(S)=1$.

Case 3. $6<\frac{3 n}{c} \leq 7<8<\frac{3 n}{b} \leq 9$.

If $\frac{27}{4}<\frac{3 n}{c} \leq 7<8<\frac{3 n}{b} \leq 9$. If $8 a<n$, let $m=8$ and $k=3$, then $\operatorname{ind}(S)=1$. If $8 a>n$, let $M=9$, then $|M e|_{n}+|M c|_{n}+|M(n-b)|_{n}+|M(n-a)|_{n}=M e+(M c-3 n)+(4 n-M b)+(2 n-M a)=$ $3 n$, and $\operatorname{ind}(S)=1$.

Next assume that $6<\frac{3 n}{c}<\frac{27}{4}<7<8<\frac{3 n}{b} \leq 9$.

If $p_{1}=5$, then $n>200 e$. We only need repeat the proof of Case 3 of Lemma 3.10 in [11]. Then $p_{3}=5$.

Subcase 3.1. $\operatorname{gcd}(n, 7)=\operatorname{gcd}(n, 11)=1$.

We infer that $7 a>n$ and $n \geq 85 p_{1}$.

If $11 b<4 n$ and $11 c>5 n$, we have $|11 e|_{n}+|11 c|_{n}+|11(n-b)|_{n}+|11(n-a)|_{n}=n$ and thus $\operatorname{ind}(S)=1$.

If $11 b>4 n$ and $11 c<5 n$, we have $|11 e|_{n}+|11 c|_{n}+|11(n-b)|_{n}+|11(n-a)|_{n}=3 n$ and thus $\operatorname{ind}(S)=1$.

If $11 b<4 n$ and $11 c<5 n$, then $\frac{n}{7}<a=c-b+e \leq \frac{5 n-e p_{3}}{11}-\frac{n+e}{3}+e \leq \frac{4 n+7 e}{33}$, so $n<10 e$, or $\frac{n}{7}<a=\frac{10}{9}(c-b)<\frac{40 n}{297}$, either of them implies a contradiction.

If $11 b>4 n$ and $11 c>5 n$, then $\frac{n}{7}<a=c-b+e \leq \frac{n-e}{2}-\frac{4 n+p_{3} e}{11}+e \leq \frac{3 n+e}{22}$, so $n<7 e$, or $\frac{n}{7}<a=c-b+e \leq \frac{n-p_{1} p_{3}}{2}-\frac{4 n+p_{1}}{11}+e \leq \frac{3 n+12 e}{22}$, so $n<84 e$, or $\frac{n}{7}<a=c-b+e \leq$ $\frac{n-p_{1} p_{3}}{2}-\frac{4 n+p_{1}}{11}+e \leq \frac{3 n+8 e}{22}$, so $n<56 e$. By Lemma 5.7, each of above implies a contradiction.

Subcase 3.2. 11|n. We infer that $8 a>n, e=11$, and $n \geq 95 e$. 
The proof is similar to Subcase 3.1.

Subcase 3.3. $7 \mid n$. We infer that $8 a>n, e=7$, and $n \geq 145 e$.

The proof is similar to Subcase 3.1.

Lemma 5.9. If the assumption is as in Proposition 2.16 and $k_{1}=2$, then $\operatorname{ind}(S)=1$.

Proof. By Lemma 2.5, it holds that $2+t<\frac{n}{c}<\frac{n}{b} \leq 3+t$ for some $t \in[0,5]$. We distinguish six cases.

Case 1. $t=0$. Then $2<\frac{n}{c}<\frac{n}{b} \leq 3$ and $4<\frac{2 n}{c} \leq 5<\frac{2 n}{b} \leq 6$.

Similar to Lemma 5.7, we infer that $6 a<n$ and hence $5 \mid n$, by Lemma 5.8 , ind $(S)=1$.

Case 2. $t=1$. Then $3<\frac{n}{c}<\frac{n}{b} \leq 4$. We infer that $a<\frac{n}{9}$ and $6<\frac{2 n}{c} \leq 7<\frac{2 n}{b} \leq 8$. Thus $7 \mid n$.

If $9<\frac{3 n}{c} \leq 10<11<\frac{3 n}{b} \leq 12$, let $m \in[10,11]$ (since $n$ can't be $5 \times 7 \times 11=385$ ) such that $\operatorname{gcd}(n, m)=1$. If and $m a<n$, let $k=3$, then $\operatorname{ind}(S)=1$. If $m a>n$, let $M=12$, we have $M e<4 a<n, n<M a<2 n$ and $3 n<M b<M c<4 n$, then $|M e|_{n}+|M c|_{n}+|M(n-b)|_{n}+$ $|M(n-a)|_{n}=M e+(M c-3 n)+(4 n-M b)+(2 n-M a)=3 n$, and $\operatorname{ind}(S)=1$.

If $9<\frac{3 n}{c} \leq 10<\frac{3 n}{b} \leq 11$, we infer that $a<\frac{n}{12}$ and $5 \mid n$. If $12<\frac{4 n}{c} \leq 13<\frac{4 n}{b} \leq \frac{44}{3}$, we infer that $13 a>n$. Otherwise, let $m=13$ and $k=4$, we have $\operatorname{gcd}(n, 13)=1$ (otherwise, $n=5 \times 7 \times 13=455<1000)$, then $\operatorname{ind}(S)=1$. Let $M=22$, it is easy to check that $\operatorname{gcd}(n, M)=1$. If $M c<7 n$, we have $M e<n, n<M a<2 n$ and $6 n<M b<M c<7 n$, and $|M e|_{n}+|M c|_{n}+$ $|M(n-b)|_{n}+|M(n-a)|_{n}=M e+(M c-6 n)+(7 n-M b)+(2 n-M a)=3 n, \operatorname{thus} \operatorname{ind}(S)=1$. If $M c>7 n$, we have $|M e|_{n}<\frac{n}{2},|M c|_{n}<\frac{n}{2}$ and $|M(n-a)|_{n}<\frac{n}{2}$, then $\operatorname{ind}(S)=1$.

If $10<\frac{3 n}{c} \leq 11<\frac{3 n}{b} \leq 12$, we infer that $a<\frac{n}{15}$ and $11 \mid n$. If $\frac{4 n}{c} \leq 15<\frac{4 n}{b}$, then $\operatorname{gcd}(15, n)=$ 1 and $15 a<n$. Let $m=15$ and $k=4$, we have $\operatorname{ind}(S)=1$. So $13<\frac{40}{3}<\frac{4 n}{c} \leq 14<\frac{4 n}{b} \leq 15$, and we infer that $a<\frac{n}{22}$. Let $M=25$, we have $\operatorname{gcd}(n, M)=1$ and

$$
6 n+\frac{2 n}{3}=\frac{100 n}{15}<M b<\frac{75 n}{11}=6 n+\frac{9 n}{11}<7 n<7 n+\frac{n}{7}=\frac{50 n}{7}<M c<\frac{15 n}{2}=7 n+\frac{n}{2} .
$$

Then $|M e|_{n}<\frac{n}{2},|M c|_{n}<\frac{n}{2}$ and $|M(n-b)|_{n}<\frac{n}{2}$, thus $\operatorname{ind}(S)=1$.

Case 3. $t=2$. Then $4<\frac{n}{c}<\frac{n}{b} \leq 5$. We infer that $a<\frac{n}{15}$ and $8<\frac{n}{c}<9<\frac{n}{b} \leq 10$. Let $m=9$ and $k=2$, then $\operatorname{ind}(S)=1$.

Case 4. $t=3$. Then $5<\frac{n}{c}<\frac{n}{b} \leq 6,10<\frac{2 n}{c} \leq 11<\frac{2 n}{b} \leq 12$ and $a<\frac{n}{22}$. If $\operatorname{gcd}(n, 11)=1$, let $m=11$ and $k=2$, we have $\operatorname{ind}(S)=1$. If $\frac{3 n}{c}<16<\frac{3 n}{b}$, let $m=16$ and $k=3$, then $\operatorname{ind}(S)=1$. Otherwise, we have $a<\frac{4}{3}(a-e)=\frac{4}{3}(c-b)<\frac{4}{3}\left(\frac{3 n}{16}-\frac{n}{6}\right)=\frac{n}{36}$, and $16<\frac{3 n}{c} \leq 17<\frac{3 n}{b}<18$. Similarly, $\operatorname{gcd}(n, 17)>1$ and $27<\frac{5 n}{c}<\frac{5 n}{b} \leq 30$.

If $27<\frac{5 n}{c} \leq 28<\frac{5 n}{b} \leq 29$, we have $a<\frac{4 \times 2 \times 5 n}{3 \times 27 \times 29}$, and $b>\frac{5 n}{29}>\frac{5}{29} \frac{3 \times 27 \times 29 a}{4 \times 2 \times 5}=\frac{81 a}{8}>10 a$, which contradicts to $s \leq 9$.

If $28<\frac{5 n}{c} \leq 29<\frac{5 n}{b} \leq 30$, we have $a<\frac{4 \times 2 \times 5 n}{3 \times 28 \times 30}$, and $b>\frac{n}{6}>\frac{1}{6} \frac{3 \times 28 \times 30 a}{4 \times 2 \times 5}=\frac{21 a}{2}>10 a$, which contradicts to $s \leq 9$.

If $27<\frac{5 n}{c} \leq 28<29<\frac{5 n}{b} \leq 30$, then there exists $m$ between $\frac{5 n}{c}$ and $\frac{5 n}{b}(m=28$ or $m=29)$ such that $\operatorname{gcd}(n, m)=1$. Let $k=5$, we have $\operatorname{ind}(S)=1$. 
Case 5. $t=4$. Then $6<\frac{n}{c}<\frac{n}{b} \leq 7$. We infer that $12<\frac{2 n}{c} \leq 13<\frac{2 n}{b} \leq 14, a<\frac{n}{31}$ and thus $\operatorname{gcd}(n, 13)>1$.

If $18<\frac{3 n}{c} \leq 19<20<\frac{3 n}{b} \leq 21$, we infer that $n=5 \times 13 \times 19$. If $\frac{5 n}{c} \leq 31<\frac{5 n}{b}$, let $m=31$ and $k=5$, we have $\operatorname{gcd}(n, m)=1$, then $\operatorname{ind}(S)=1$. Otherwise, $31<\frac{5 n}{c}<\frac{5 n}{b} \leq 35$, hence $a<\frac{4}{3} \times\left(\frac{5 n}{31}-\frac{5 n}{35}\right)=\frac{n}{40}$. Then, for any integer $m$ between $\frac{5 n}{c}$ and $\frac{5 n}{b}$, we have $\operatorname{gcd}(n, m)=1$ and $m a<n$, hence $\operatorname{ind}(S)=1$.

If $18<\frac{3 n}{c} \leq 19<\frac{3 n}{b} \leq 20$, we infer that $a<\frac{n}{45}$. If $\frac{5 n}{c}<32<\frac{5 n}{b}$, let $m=32$ and $k=5$, then we have done. Otherwise, $30<\frac{5 n}{c} \leq \frac{95}{3}<\frac{5 n}{b}<32$, thus we infer that $a<\frac{n}{72}$ and $\frac{b}{a}>\frac{5 \times 72}{32}=\frac{45}{4}>10$, which contradicts to $s \leq 9$.

If $19<\frac{3 n}{c} \leq 20<\frac{3 n}{b} \leq 21$, we infer that $a<\frac{n}{49}$ and $\operatorname{gcd}(n, 20)>1$. If $27<\frac{4 n}{b}$, then $\frac{4 n}{c} \leq \frac{80}{3}<27<\frac{4 n}{b}$, let $m=27$ and $k=4$, we have $\operatorname{ind}(S)=1$. Otherwise, $\frac{76}{3} \frac{4 n}{c}<\frac{4 n}{b}<27$, we infer that $a<\frac{4 \times 5 n}{19 \times 81}$, and $\frac{b}{a}>\frac{4}{27} \times \frac{19 \times 81}{20}=\frac{57}{5}>10$, which contradicts to $s \leq 9$.

Case 6. $t=5$. Then $7<\frac{n}{c}<\frac{n}{b} \leq 8$. We infer that $14<\frac{2 n}{c} \leq 15<\frac{2 n}{b} \leq 16, a<\frac{n}{42}$ and thus $\operatorname{gcd}(n, 15)>1$.

If $21<\frac{3 n}{c} \leq 22<23<\frac{3 n}{b} \leq 24$, we infer that $n=5 \times 11 \times 23$. If 29 or 31 belongs to $\left[\frac{4 n}{c}, \frac{4 n}{b}\right)$, let it be $m$ and $k=4$, we have $\operatorname{gcd}(n, m)=1$, then $\operatorname{ind}(S)=1$. Otherwise, $29<\frac{4 n}{c} \leq 30<\frac{4 n}{b} \leq 31$, hence $a<\frac{4}{3} \times\left(\frac{4 n}{29}-\frac{4 n}{31}\right)=\frac{32 n}{3 \times 29 \times 31}$, and $\frac{b}{a}>\frac{4}{31} \times \frac{3 \times 29 \times 31}{32}=\frac{87}{8}>10$, which contradicts to $s \leq 9$.

If $21<\frac{3 n}{c} \leq 22<\frac{3 n}{b} \leq 23$, we infer that $a<\frac{n}{60}$. If $\frac{5 n}{c}<36<\frac{5 n}{b}$, let $m=36$ and $k=5$, then we have done. Otherwise, $36<\frac{5 n}{c} \leq 37<\frac{5 n}{b} \leq \frac{115}{3}$, thus we infer that $a<\frac{7 n}{23 \times 27}$ and $\frac{b}{a}>\frac{23 \times 27}{7} \times \frac{3}{23}=\frac{81}{3}>10$, which contradicts to $s \leq 9$.

If $22<\frac{3 n}{c} \leq 23<\frac{3 n}{b} \leq 24$, we infer that $a<\frac{n}{66}$ and $\operatorname{gcd}(n, 23)>1$. If $\frac{5 n}{c} \leq 37$, then $\frac{5 n}{c} \leq 37<38<\frac{115}{3}<\frac{5 n}{b} \leq 40$. There exists $m \in[37,38]$ such that $\operatorname{gcd}(m, n)=1$, let $k=5$, then $\operatorname{ind}(S)=1$. Similarly, $\frac{5 n}{c} \leq 38<39<\frac{5 n}{b} \leq 40$ implies $\operatorname{ind}(S)=1$. If $37<\frac{5 n}{c} \leq 38<\frac{5 n}{b} \leq 39$ or $38<\frac{5 n}{c} \leq 39<\frac{5 n}{b} \leq 40$, we infer that $b>10 a$, a contradiction.

\section{Acknowledgements}

The second author is thankful to prof. Yuanlin Li and prof. Jiangtao Peng for their useful discussion and valuable comments.

\section{REFERENCES}

[1] S.T. Chapman, M. Freeze, and W.W Smith, Minimal zero sequences and the strong Davenport constant, Discrete Math. 203(1999), 271-277.

[2] S.T. Chapman, and W.W Smith, A characterization of minimal zero-sequences of index one in finite cyclic groups, Integers 5(1)(2005), Paper A27, 5p.

[3] W. Gao, Zero sums in finite cyclic groups, Integers 0 (2000), Paper A14, 9p.

[4] W. Gao and A. Geroldinger, On products of k atoms, Monatsh. Math. 156 (2009), 141-157.

[5] W. Gao, Y. Li, J. Peng, P. Plyley and G. Wang On the index of sequences over cyclic groups (English), Acta Arith. 148, No. 2, (2011) 119-134

[6] A. Geroldinger, On non-unique factorizations into irreducible elements. II, Number Theory, Vol II Budapest 1987, Colloquia Mathematica Societatis Janos Bolyai, vol. 51, North Holland, 1990, 723-757.

[7] A. Geroldinger, Additive group theory and non-unique factorizations, Combinatorial Number Theory and Additive Group Theory (A. Geroldinger and I. Ruzsa, eds.), Advanced Courses in Mathematics CRM Barcelona, Birkhäuser, 2009, pp. 1-86. 
[8] A. Geroldinger and F. Halter-Koch, Non-Unique Factorizations. Algebraic, Combinatorial and Analytic Theory, Pure and Applied Mathematics, Vol. 278, Chapman \& Hall/CRC, 2006.

[9] D. Kleitman and P. Lemke, An addition theorem on the integers modulo n, J. Number Theory 31(1989),335-345.

[10] Y. Li and J. Peng, Minimal zero-sum sequences of length five over finite cyclic groups, Ars Combinatoria, to appear.

[11] Y. Li and J. Peng, Minimal zero-sum sequences of length four over finite cyclic groups II, International Journal of Number Theory, to appear

[12] Y. Li, C. Plyley, P. Yuan and X. Zeng, Minimal zero sum sequences of length four over finite cyclic groups, Journal of Number Theory. 130 (2010), 2033-2048.

[13] V. Ponomarenko, Minimal zero sequences of finite cyclic groups, Integers 4(2004), Paper A24, 6p.

[14] S. Savchen and F. Chen, Long zero-free sequences in finite cyclic groups, Discrete Math. 307(2007), 2671-2679.

[15] X. Xia and P. Yuan, Indexes of insplitable minimal zero-sum sequences of length $l\left(C_{n}\right)-1$, Discrete Math., to appear.

[16] P. Yuan, On the index of minimal zero-sum sequences over finite cyclic groups, J. Combin. Theory Ser. A114(2007), 1545-1551.

[17] P. Yuan and X. Zeng, Indexes of long zero-sum free sequences over cyclic groups, Eur. J. Comb. 32(2011), 1213-1221.

[18] D. J. Grynkiewicz, Structural Additive Theory, Developments in Mathematics, to appear, Springer, 2013.

[19] L. Xia, On the index-conjecture of length four minimal zero-sum sequences, International Journal of Number Theory, to appear. 